\title{
Efficacy of Immunobiologic and Small Molecule Inhibitor Drugs for Psoriasis: A Systematic Review and Meta-Analysis of Randomized Clinical Trials
}

\author{
André Vicente Esteves de Carvalho ${ }^{1,2}$ - Rodrigo Pereira Duquia ${ }^{1,2}$. \\ Bernardo Lessa Horta ${ }^{3}$ - Renan Rangel Bonamigo ${ }^{1,2}$
}

Published online: 12 November 2016

(C) The Author(s) 2016. This article is published with open access at Springerlink.com

\begin{abstract}
Background Psoriasis is an immune-mediated inflammatory disease for which treatment has evolved over the past few years due to the introduction of immunobiologic and small molecule inhibitor medications. A better understanding of the comparative efficacies of drugs may help doctors to choose the most appropriate treatment for patients.

Objective The aim of this study was to conduct a systematic review and meta-analysis to assess the efficacy of immunobiologic and small molecule inhibitor drugs for patients with moderate to severe psoriasis.

Data Sources The EMBASE, PUBMED, LILACS, Web of Science and ClinicalTrials.org databases were searched for trials published to 21 July 2016.

Study Selection Only randomized, double-blind, placebocontrolled clinical trials that evaluated the efficacy of immunobiologics or small molecule inhibitors for
\end{abstract}

Electronic supplementary material The online version of this article (doi:10.1007/s40268-016-0152-x) contains supplementary material, which is available to authorized users.

André Vicente Esteves de Carvalho

avecarvalho@me.com

1 Irmandade Santa Casa de Misericórdia de Porto Alegre, Ramiro Barcelos 1176/702, Porto Alegre, RS 90035-002, Brazil

2 Universidade Federal de Ciências da Saúde de Porto Alegre, Porto Alegre, RS, Brazil

3 Universidade Federal de Pelotas, Pelotas, RS, Brazil moderate to severe plaque-type psoriasis were selected by two independent authors. No restrictions were used.

Data Extraction and Synthesis Two authors independently extracted the data and a random-effects model meta-analysis was performed.

Main Outcomes and Measures The Psoriasis Area and Severity Index (PASI) 75 was considered the primary outcome, measured at the primary endpoint of each study. Results Thirty-eight studies were included in our analysis. The overall pooled effect favored biologics and small molecule inhibitors over placebo (risk difference [RD] $0.59,95 \%$ confidence interval [CI] 0.58-0.60). Ixekizumab at a dose of $160 \mathrm{mg}$ on week 0 and then every 2 weeks (RD 0.84, 95\% CI 0.81-0.88), brodalumab $210 \mathrm{mg}$ (RD 0.79, 95\% CI 0.76-0.82), infliximab $5 \mathrm{mg} / \mathrm{kg}$ (RD 0.76, 95\% CI 0.73-0.79), and secukinumab $300 \mathrm{mg}$ (RD 0.76, 95\% CI 0.71-0.81) showed a greater chance of response (PASI 75) when compared with placebo.

Limitations The methodology of a traditional meta-analysis does not allow for drugs to be ranked. Included studies used short-term endpoints (10-16 weeks) to evaluate the primary outcome, therefore long-term efficacy could not be determined.

Conclusions and Relevance The anti-IL-17 drugs brodalumab, ixekizumab and secukinumab showed an equal or greater chance of helping patients achieve a $75 \%$ improvement on PASI compared with other reviewed drugs. 


\section{Key Points}

Anti-tumor necrosis factor and anti-interleukin (IL)$12 / 23$ have been shown to be effective in treating patients with moderate to severe psoriasis.

Anti-IL-17 drugs showed an equal or greater chance of leading patients to a $75 \%$ improvement when compared with other biologics/small molecule inhibitors.

Ixekizumab showed higher efficacy among FDAapproved drugs when a 90 or $100 \%$ improvement over the baseline Psoriasis Area and Severity Index was analyzed.

\section{Introduction}

Psoriasis is a chronic, immune-mediated inflammatory disease, where an intricate immune process, mainly driven by the T-helper (Th) 1/Th17 branch of the immune system, leads to persistent inflammation [1-3].

The treatment of psoriasis has been revolutionized by the introduction of biologic and small molecule inhibitor targeted therapy. Several of these therapies have been released and are available for general use, such as infliximab [4], adalimumab [5], ustekinumab [6], apremilast [7], etanercept [8], ixekizumab [9], and secukinumab [10], while others are in phase II or later trials, e.g. brodalumab [11], guselkumab [12], certolizumab pegol [13], and tofacitinib [14]. On the other hand, studies on the efficacy of briakinumab were halted because of safety concerns during phase III trials [15].

Schmitt et al. [16] recently carried out a meta-analysis that included studies that evaluated systemic treatments for psoriasis (biologics or not) published before October 2012. This review did not include anti-IL-17 drugs, and infliximab $5 \mathrm{mg} / \mathrm{kg}$ was superior to ustekinumab, adalimumab and etanercept. Xiong et al. published a systematic review that only included secukinumab, one of the anti-IL-17 biologic drugs, and concluded that anti-IL-17 drugs would be more efficacious than currently available biologics [17]. Also in 2015, Chen et al. performed a meta-analysis comparing only anti-IL-17 drugs, and reported a greater chance of response of brodalumab $140 \mathrm{mg}$, followed by ixekizumab $25 \mathrm{mg}$ and secukinumab $150 \mathrm{mg}$ [18].

As new drugs have emerged in the last few years $[9,11,13,14]$, it is important to update previous reviews to provide the best evidence on the efficacy of recent treatments for psoriasis. This study aimed to systematically review the evidence on the efficacy of biologic and small molecule inhibitor drugs for the treatment of moderate to severe psoriasis

\section{Methods}

This systematic review and meta-analysis was conducted using the recommendations of the Cochrane Initiative, and reported using the Preferred Reporting Items for Systematic Reviews and Meta-Analyses (PRISMA) statement [19].

\subsection{Search Strategy/Databases Searched/Eligibility Criteria}

The research question ("What is the efficacy, measured by the improvement of $75 \%$ over baseline Psoriasis Area and Severity Index (PASI), of biologic and small molecule inhibitor drugs for moderate to severe psoriasis patients when compared to placebo?") was formulated using the PICO method (Population, Intervention, Comparator, Outcome). The EMBASE, PUBMED, LILACS, Web of Science and ClinicalTrials.org databases were searched for double-blind, randomized, placebo-controlled clinical trials (RCTs) published to 21 July 2016. Search strategies involved use of the terms "psoriasis AND (abatacept OR apremilast OR CC-10004 OR adalimumab OR D2E7 OR briakinumab OR ABT-480 OR brodalumab OR certolizumab OR etanercept OR TNF Fc OR fezakizumab OR golimumab OR guselkumab OR CNTO1959 OR infliximab OR ixekizumab OR secukinumab OR Ly-2439821 OR sifalimumab OR siplizumab OR tasocitinib OR tofacitinib OR ustekinumab OR CNTO-1275 OR AbGn-168 OR RG4934 OR APG-2305 OR MK-3222)'. Studies published online or in print, or studies in press were reviewed. Although we considered all languages eligible for the review, only studies published in English were found to be relevant.

Initially, duplicate studies were excluded and two researchers (AVEC and RPD) independently reviewed the titles and abstracts to exclude those studies that were clearly irrelevant. The reviewers then evaluated the full text of the remaining manuscripts and relevant articles were identified. Disagreements were solved by consensus. Randomized clinical trials were eligible for inclusion in the present review if they fulfilled the following criteria: human-based, double-blind, randomized, placebo-controlled clinical trials that evaluated adult patients and used the Psoriasis Area and Severity Index (PASI) as a measurement for psoriasis severity. Phase II studies were only included if the studied drugs or doses were identified in further phase III studies, or if drugs or doses were already 
approved by the US FDA. In the case of studies with multiple study arms, including approved and non-approved drugs or doses, only the arms containing approved drugs/doses were included in the meta-analysis. Head-tohead studies without a placebo arm were excluded from the analysis, and studies that evaluated the improvement of psoriatic arthritis as a primary outcome were also excluded. The reference lists of the articles included in the review were searched for additional studies.

The primary efficacy outcome was the number of patients who experienced a $75 \%$ improvement in disease status, measured by the PASI (PASI 75), at the time of the primary efficacy assessment. Secondary outcomes were 90\% improvement (PASI 90) and 100\% improvement (PASI 100) in disease status.

\subsection{Data Extraction}

Using a standardized protocol [20] entirely based on the Cochrane handbook for systematic reviews and interventions, reviewers extracted the following items from each study: authors; year of publication; intervention and comparator; total number of patients randomized; trial duration; mean disease duration; mean age of patients; mean baseline PASI; number of patients achieving PASI 75, 90 and 100; and prior use of biologic or concomitant medications. Effect estimates were extracted with 95\% confidence intervals (CI). Any disagreement was also solved by consensus. It was decided not to use any quality assessment of the studies (i.e. JADAD), and to evaluate its impact on the estimated pooled effect using meta-regression/sensitivity analysis. Data regarding randomization, blinding, and complete reporting of the study results were also extracted to evaluate the risk of bias [20].

\subsection{Statistical Analysis}

Effect measures were reported as the pooled risk difference (RD), and an $\mathrm{RD}>0$ denoted that those subjects who received the 'new drug' showed a higher risk of presenting the outcome. The overall pooled effect of any treatment versus placebo was estimated by running a separate analysis, with all treatment patients gathered in one group and all placebo patients in another group, to avoid the unit-ofanalysis error. The number needed to treat (NNT) was also calculated.

Heterogeneity among studies was assessed using the Q-test and $I^{2}$, and a random-effects model was used. Sensitivity analysis was performed excluding phase II studies when heterogeneity was found to be $>50 \%$. A funnel plot and Egger's test were used to investigate publication bias. We performed meta-regression to assess the influence of mean baseline PASI, previous use of biologics, and duration of the disease on the heterogeneity among studies.

Meta-analysis using data extracted from the studies was performed using STATA v.14 software for Mac (StataCorp LP, College Station, TX, USA). Forest plots, funnel plots, and risk of bias assessment graphs were developed using Review Manager Version 5.3. (Copenhagen: The Nordic Cochrane Centre, The Cochrane Collaboration, 2014).

\section{Results}

Overall, 9544 records were identified through a database search, with 5 additional records identified through a search of the bibliographical references of published meta-analyses. After removing duplicates, 6513 records were screened and 6181 were excluded (3822 were not RCTs, 2039 did not pertain to psoriasis, 122 pertained to drugs not encompassed in this review, and 198 were additional duplicates).

Among the 332 articles selected for full-text review, 292 were excluded for the following reasons: 201 were not RCTs, 16 were RCTs that did not use placebo as the comparator, 45 due to the lack of PASI as the primary outcome, 5 were studies in the pediatric population, 4 were phase II studies without further confirmatory phase III studies, 8 were studies with doses not approved by the FDA, 9 pertained to psoriatic arthritis, and 4 were additional duplicates (Fig. 1).

A total of 40 studies $[4,5,7-9,11,14,21-53]$ were included in the meta-analysis, providing 56 comparisons of 11 different interventions. In total, 22,884 patients were evaluated. The medications studied were adalimumab, apremilast, brodalumab, etanercept, infliximab, ixekizumab, secukinumab, tofacitinib and ustekinumab. Of the 40 studies included in the meta-analysis, 6 used a 10-week endpoint, 6 used a 16-week endpoint, and 28 used a 12-week endpoint. Primary endpoints for outcomes assessment were correlated with the induction period of the drugs and can be considered short-term therapy. All studies shared similar inclusion criteria and baseline characteristics (Table 1). Risk of bias assessment showed that high risk of bias was low among the studies (Online Resource 1 and 2).

At FDA-approved dose regimens, 1054 patients were randomized to adalimumab, 650 to apremilast, 2957 to etanercept (535-50 mg/wk, and 2422-100 mg/wk), 844 to infliximab, 1169 to ixekizumab, 691 to secukinumab, and 1678 to ustekinumab (949-45 mg, and 729-90 mg). With regard to drugs still not approved by the FDA, 2554 patients were randomized to brodalumab $(1278-140 \mathrm{mg}$, and $1276-210 \mathrm{mg}$ ) and 2197 to tofacitinib (1124-5 mg, and $1073-10 \mathrm{mg}$ ). 
Fig. 1 PRISMA statement diagram for database searches for meta-analysis of the efficacy of biologics and small molecule inhibitors for psoriasis. PRISMA Preferred Reporting Items for Systematic Reviews and MetaAnalyses, RCTs randomized controlled trials, PASI Psoriasis Area and Severity Index
9544 records identified through database search

(EMBASE, PubMed,

LILACS, Web of science and clinicaltrials.org)
5 aditional record identified

through other sources

(published meta-analysis)
6513 records after

duplicates removed
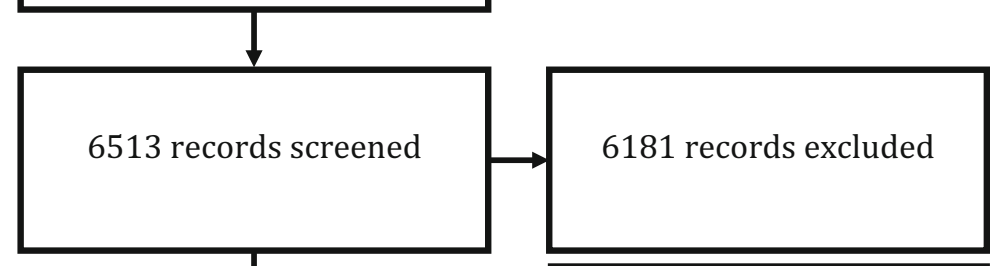

332 full-text articles assessed for eligibility

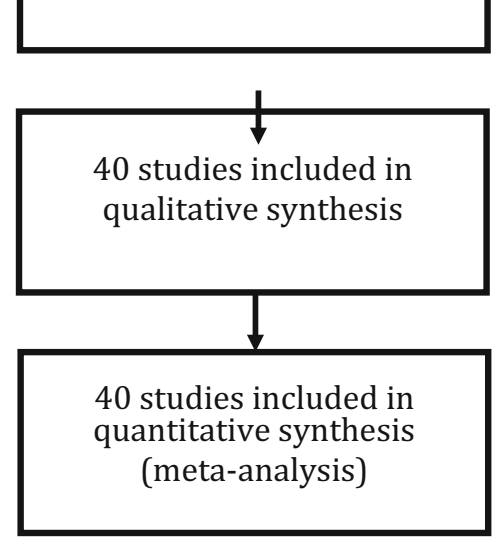

Considering PASI 75 as the primary endpoint, ixekizumab (160 mg week 0 and $80 \mathrm{mg}$ every 2 weeks) was the drug that achieved the higher RD $(0.84,95 \%$ CI 0.81-0.88), followed by brodalumab at a dose of $210 \mathrm{mg}$ (weeks $0,1,2,4,6,8$, and 10) [RD 0.79, 95\% CI
292 full-text articles excluded, with reasons

- 201 articles were not RCTs

- 16 due to lack of placebo

- 45 due to lack of PASI as primary outcome

- 5 were studies in the pediatric population

- 4 phase II studies about drugs without further development or confirmatory phase III studies

- 8 studies with drug doses not approved by the FDA

- 4 duplicates

- 9 about psoriatic arthritis
0.76-0.82). Figure 2 shows the remaining comparisons. Infliximab $5 \mathrm{mg} / \mathrm{kg}$ (RD 0.76, 95\% CI 0.73-0.79) and secukinumab $300 \mathrm{mg}$ (RD 0.76, 95\% CI 0.71-0.81) performed comparably. The overall pooled effect favored treatment when compared with placebo (RD 0.59, 95\% CI 


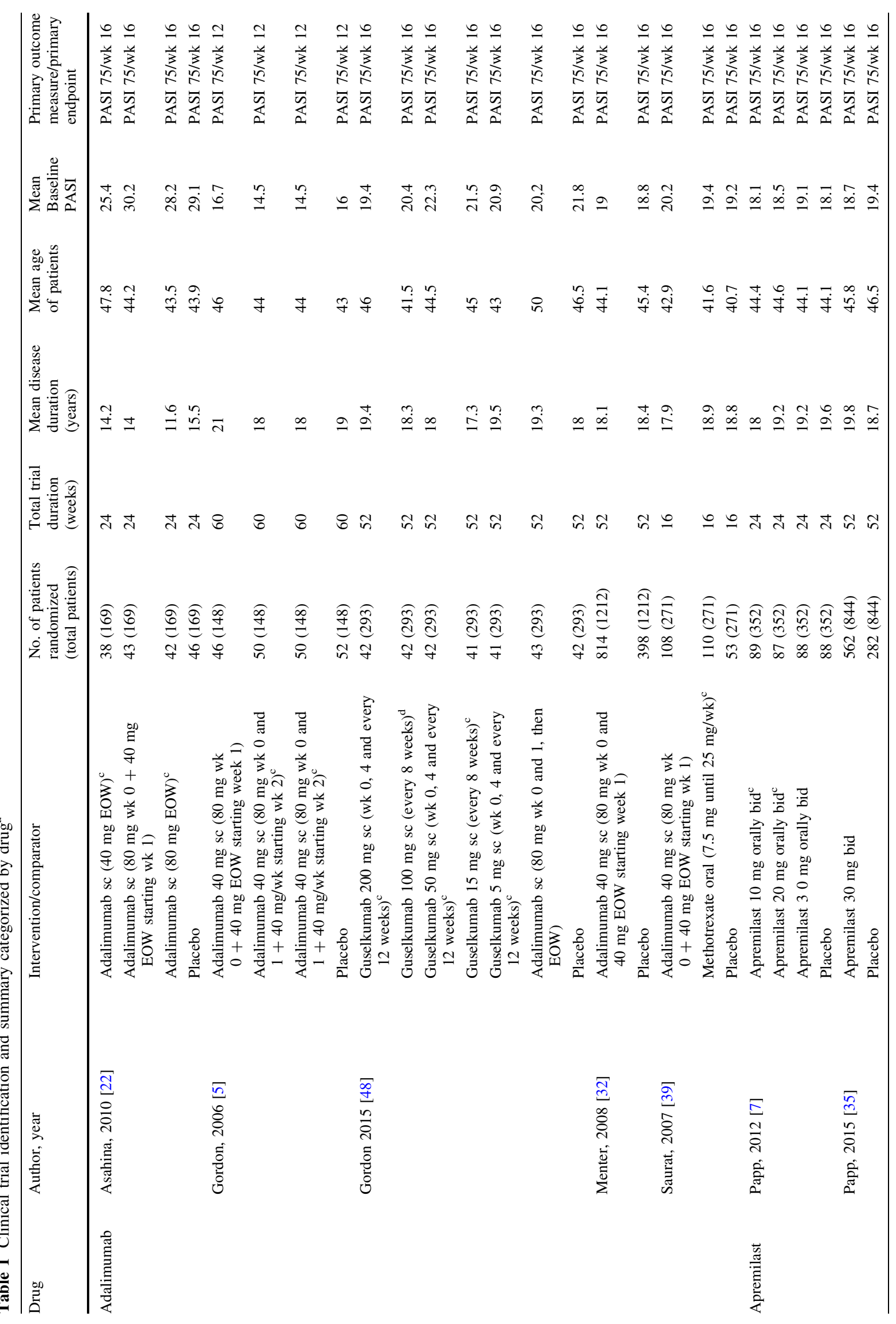




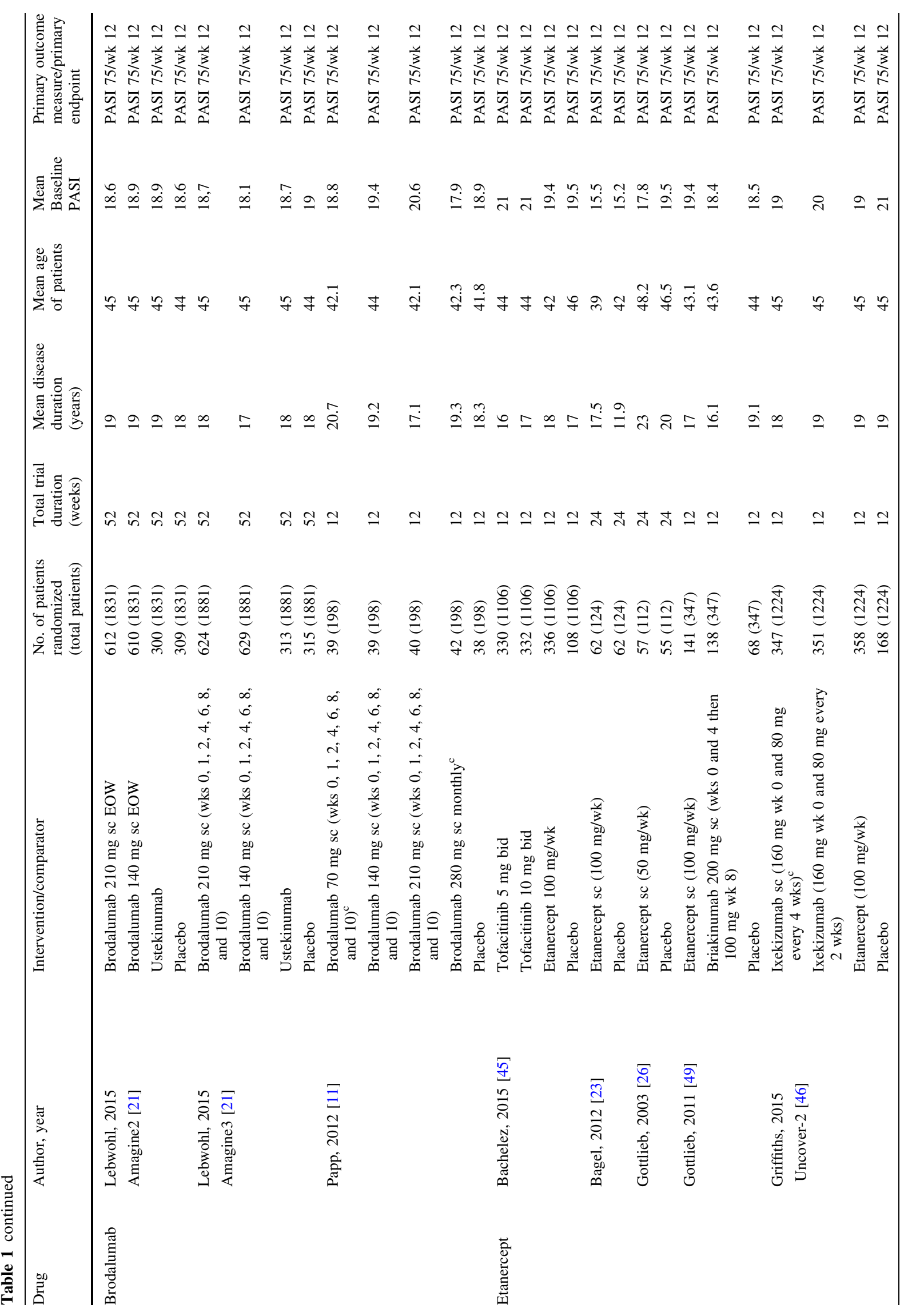




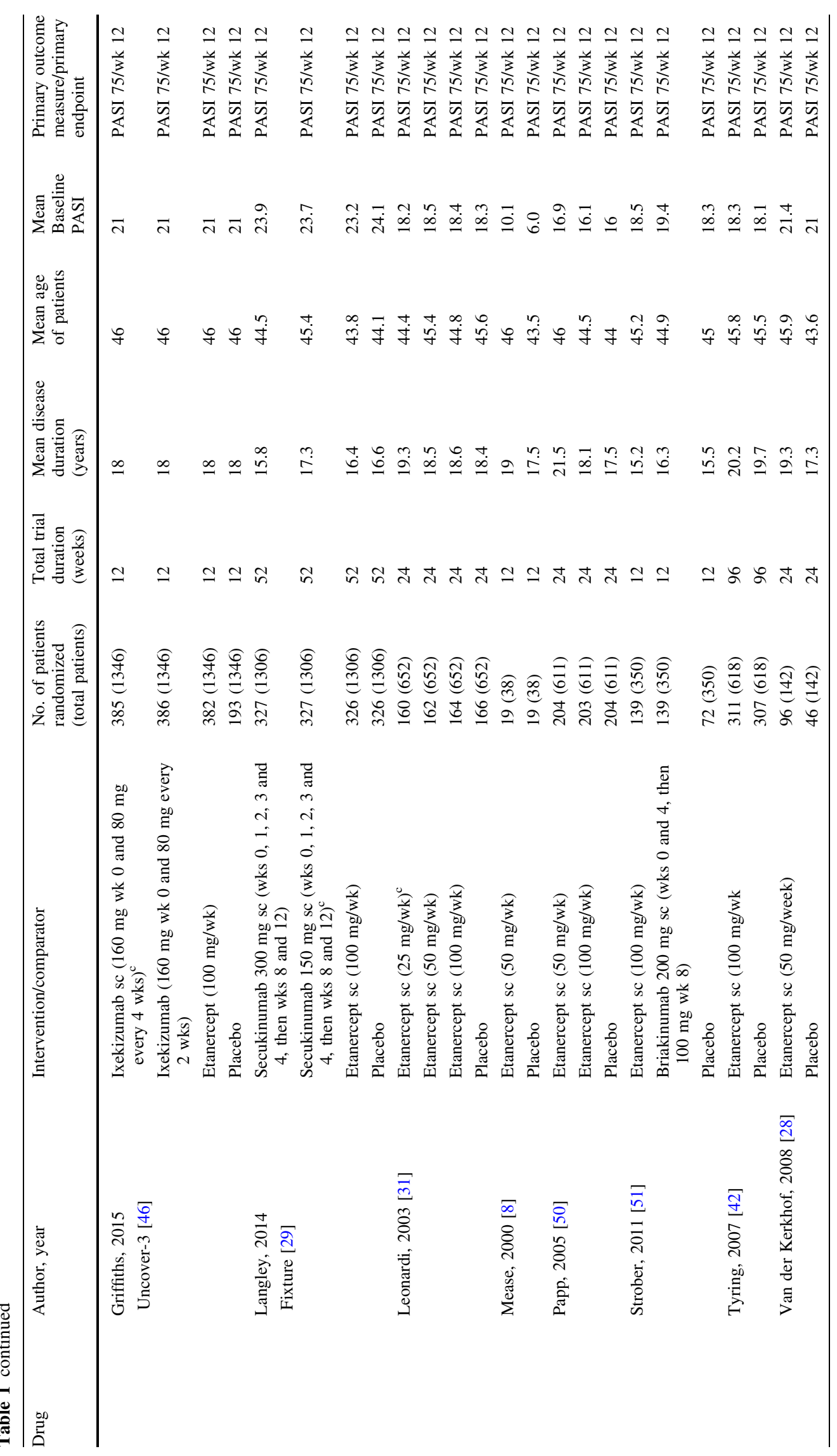




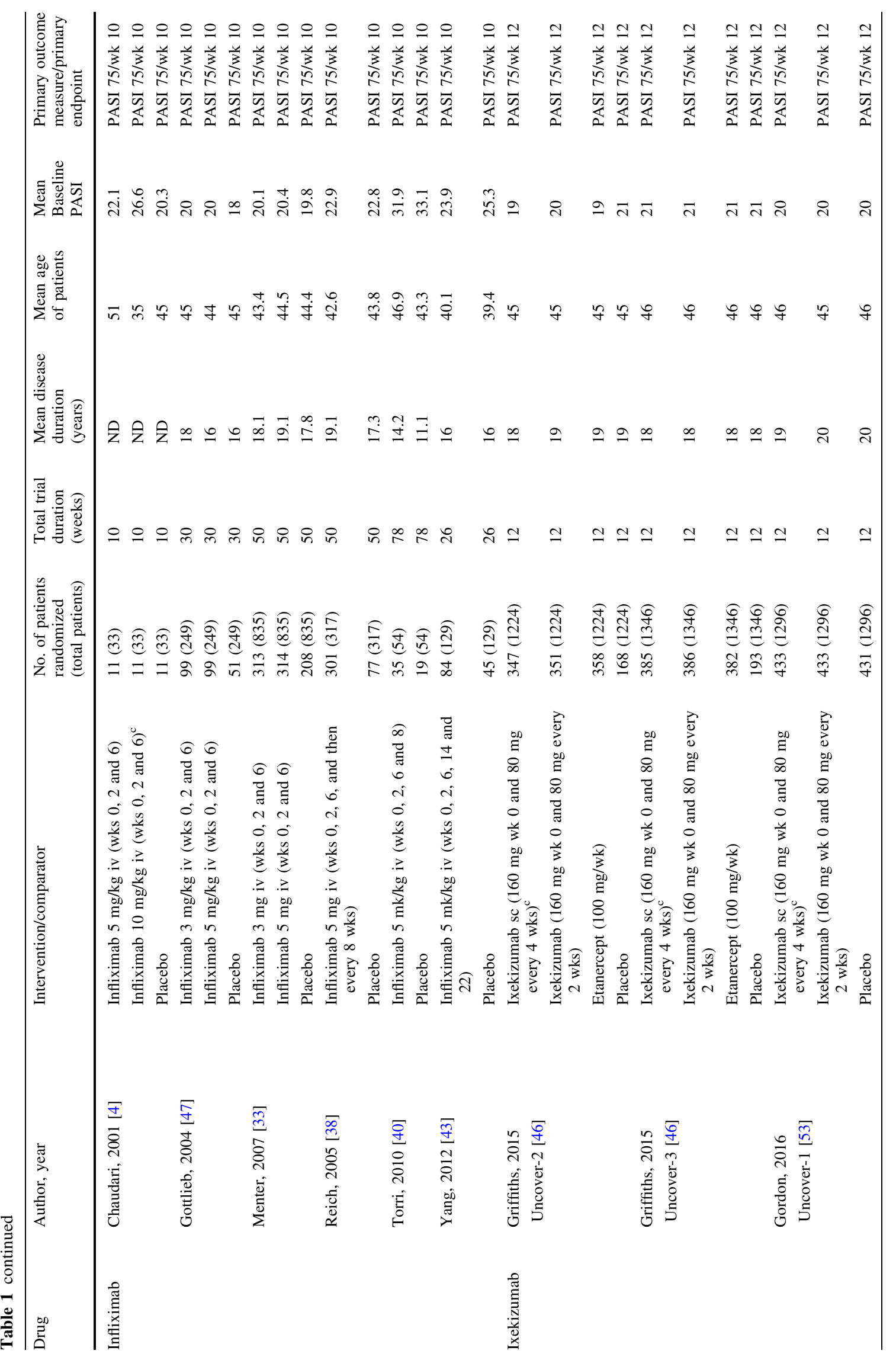




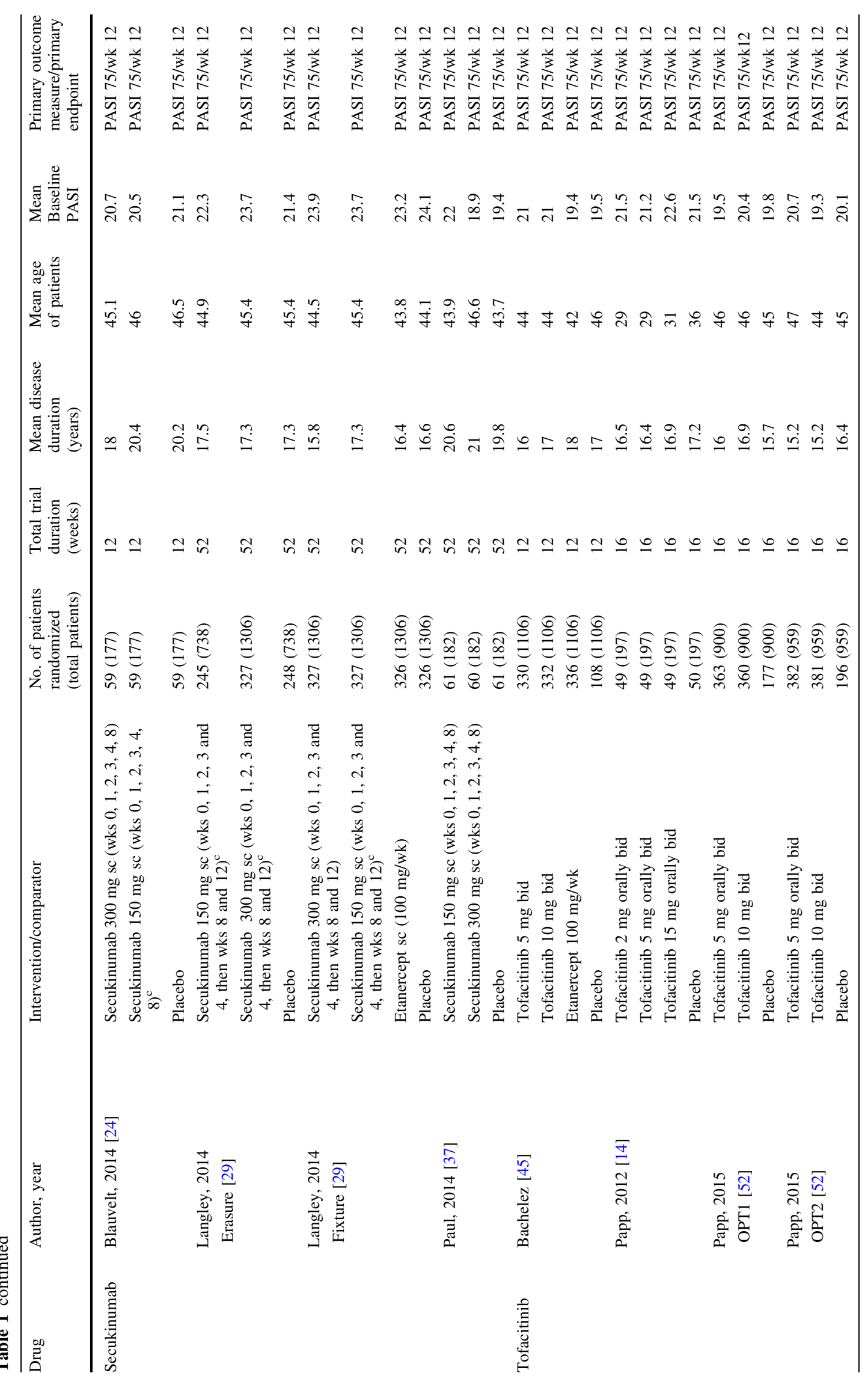




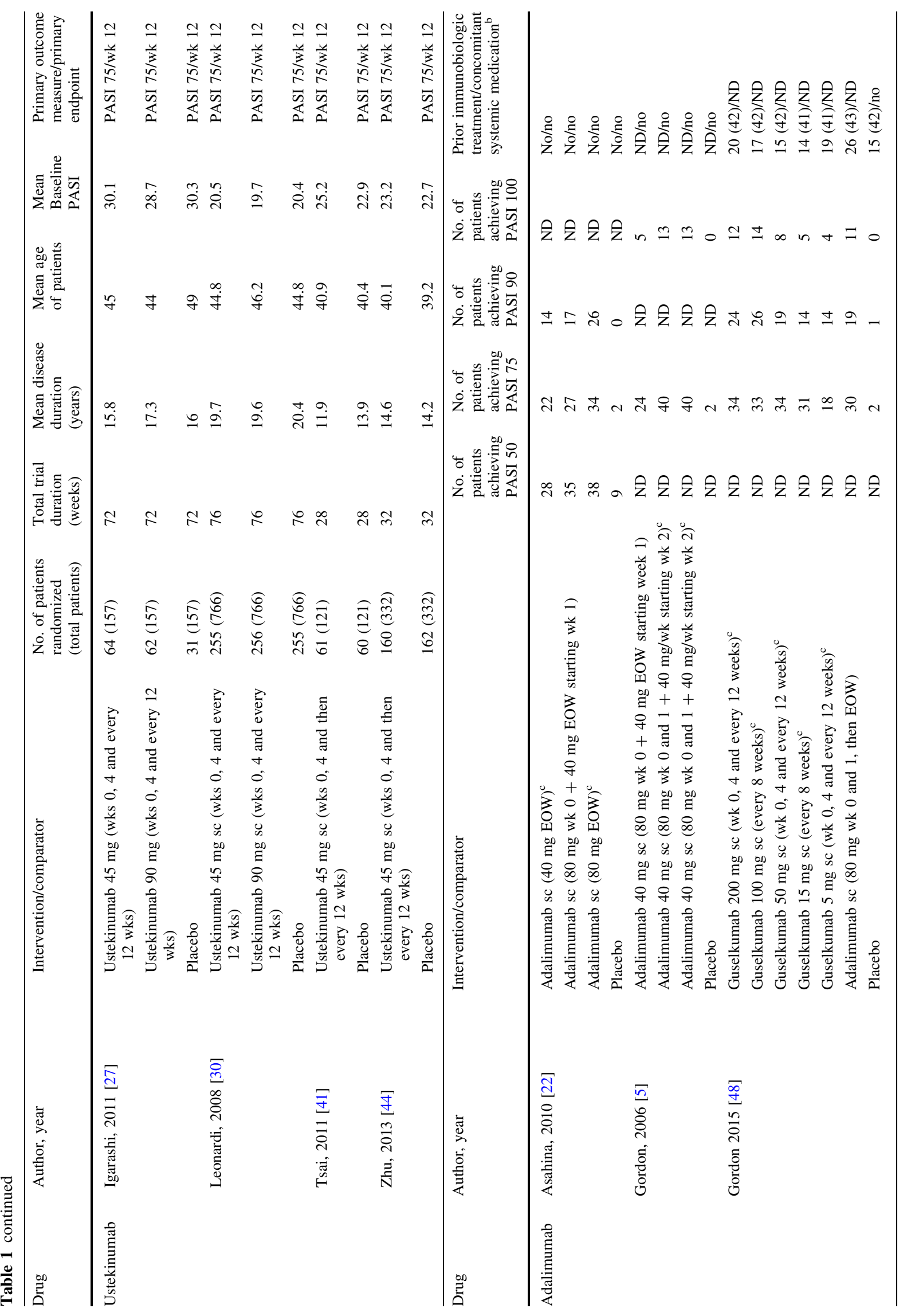




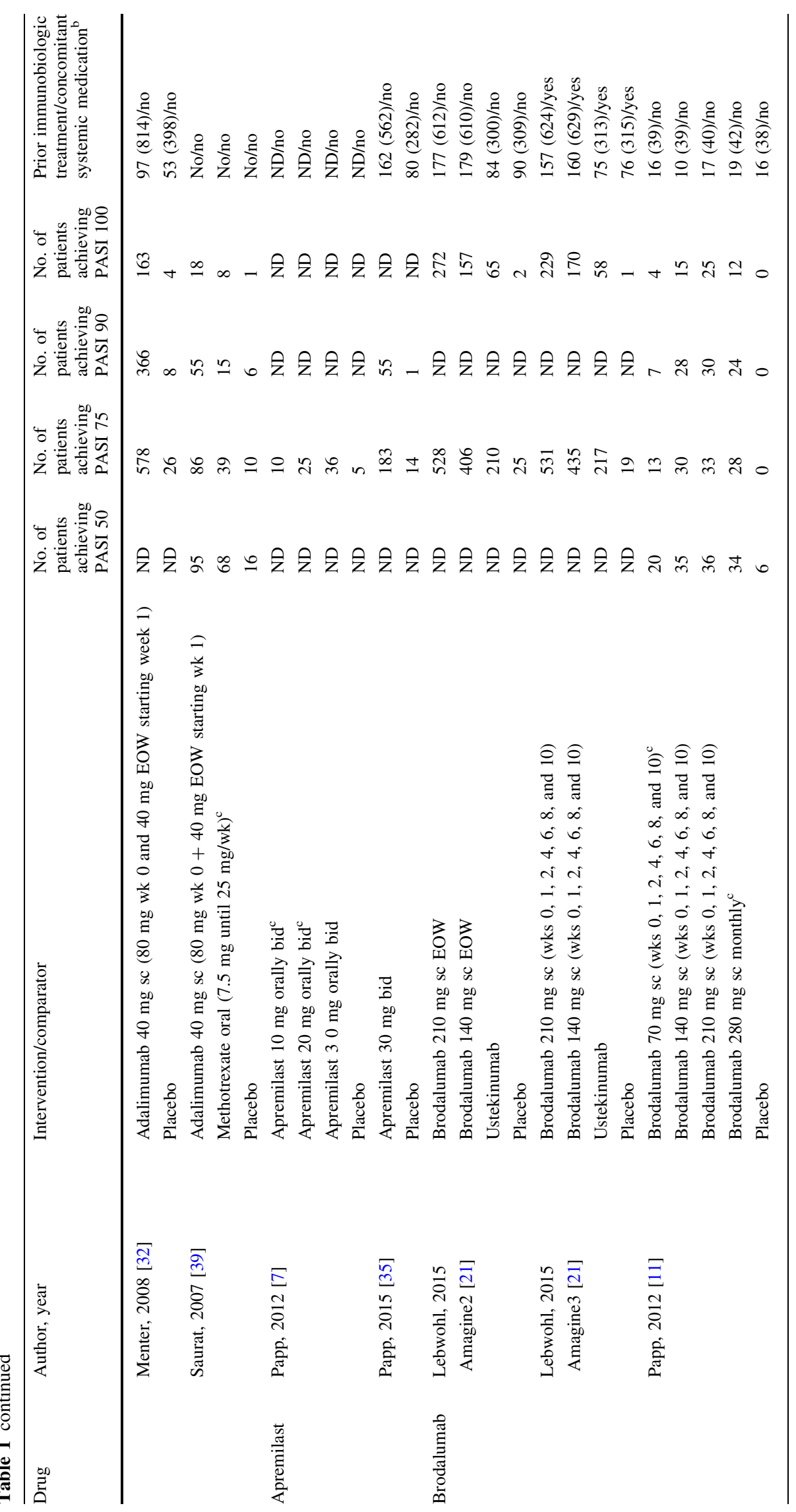




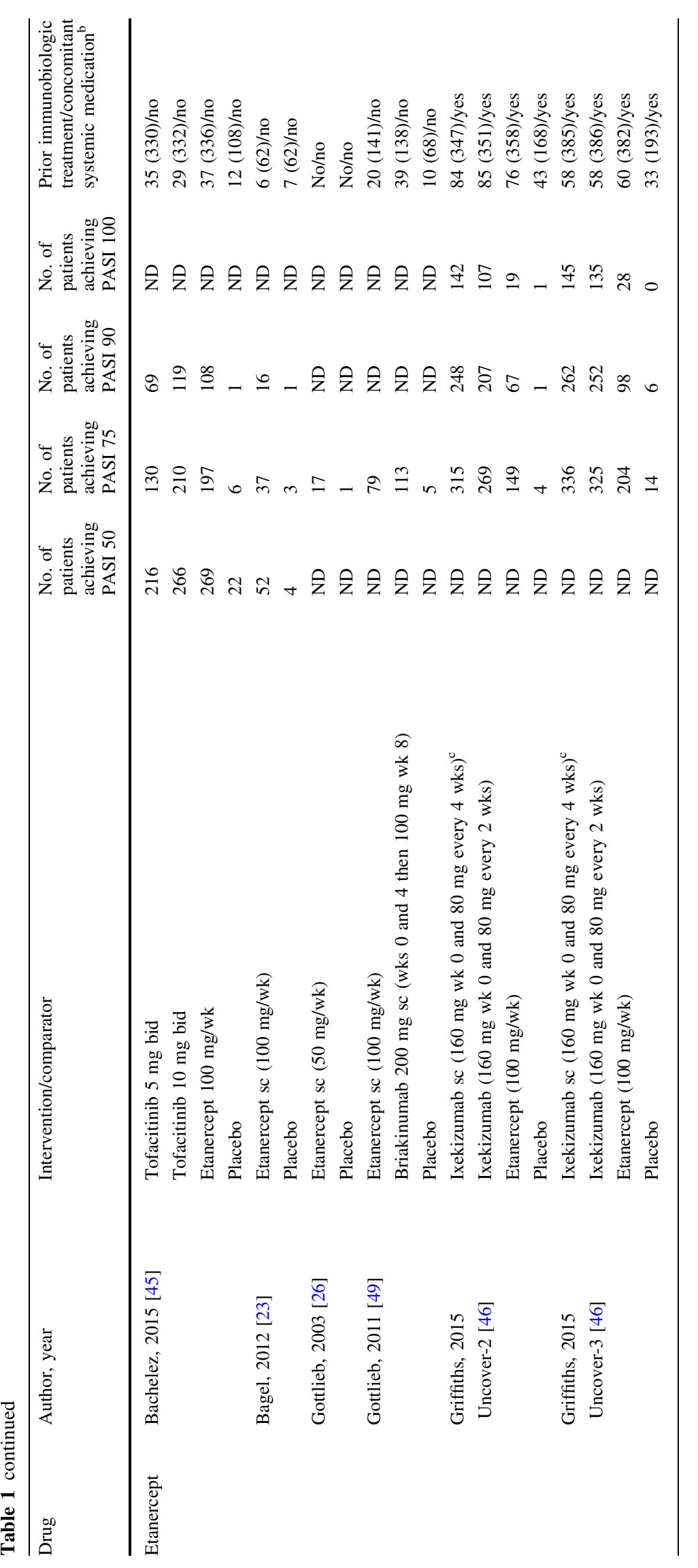




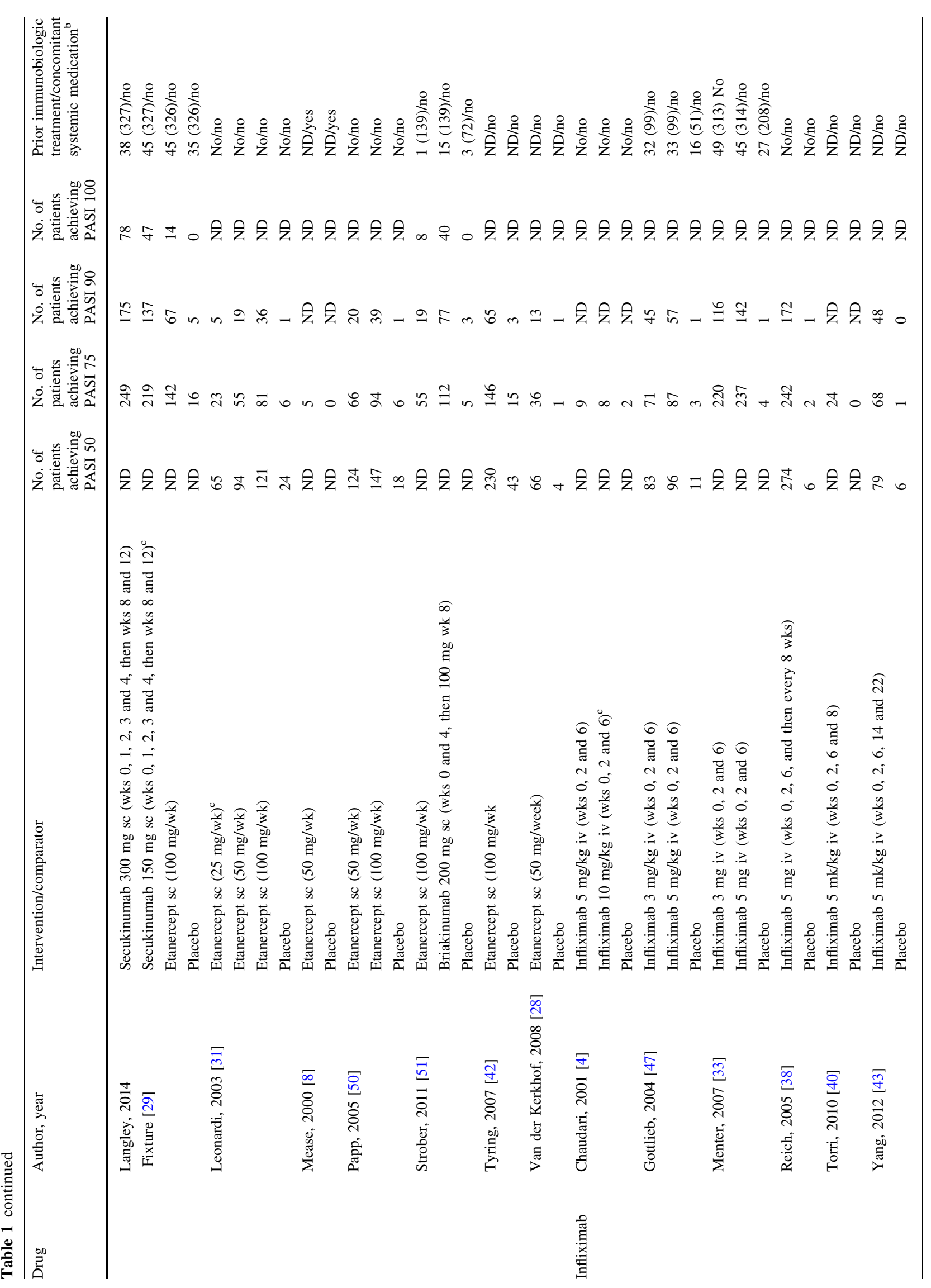




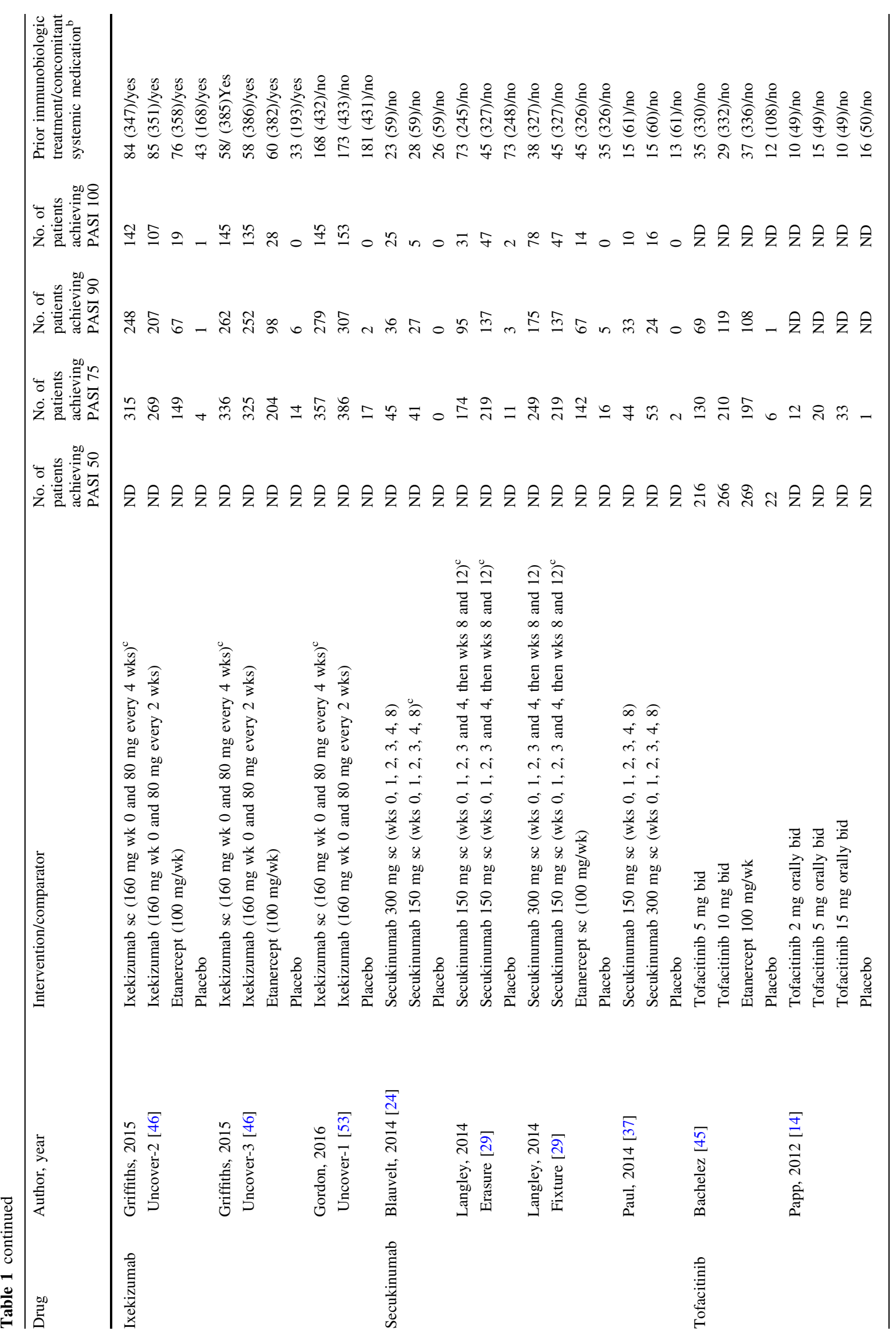




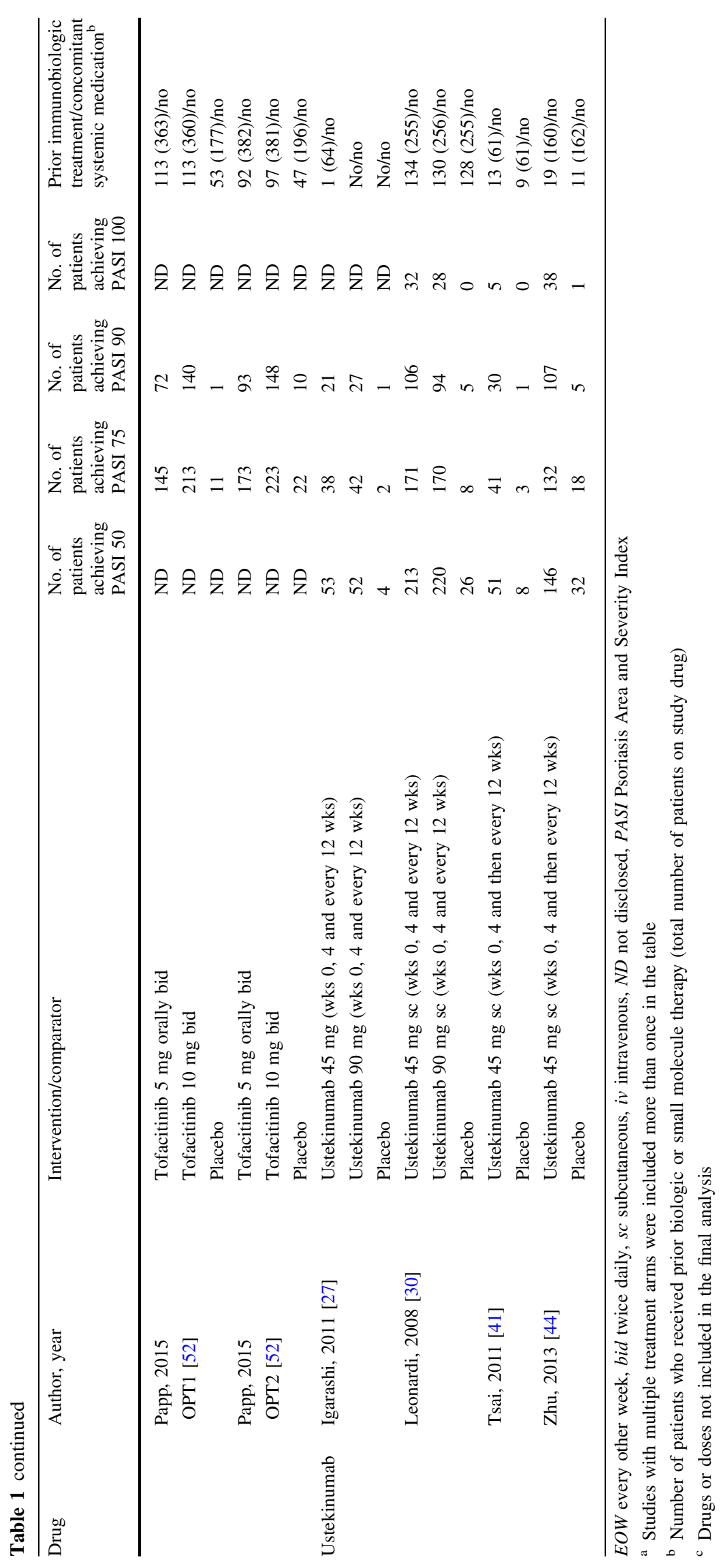


Fig. 2 Meta-analysis (randomeffects model) of the Psoriasis Area and Severity Index 75\% response rate of biologic and small molecule inhibitor therapies for moderate to severe psoriasis in randomized, placebo-controlled trials. $C I$ confidence interval, $M-H$ Mantel-Haenszel, EOW every other week, $d f$ degrees of freedom, bid twice daily

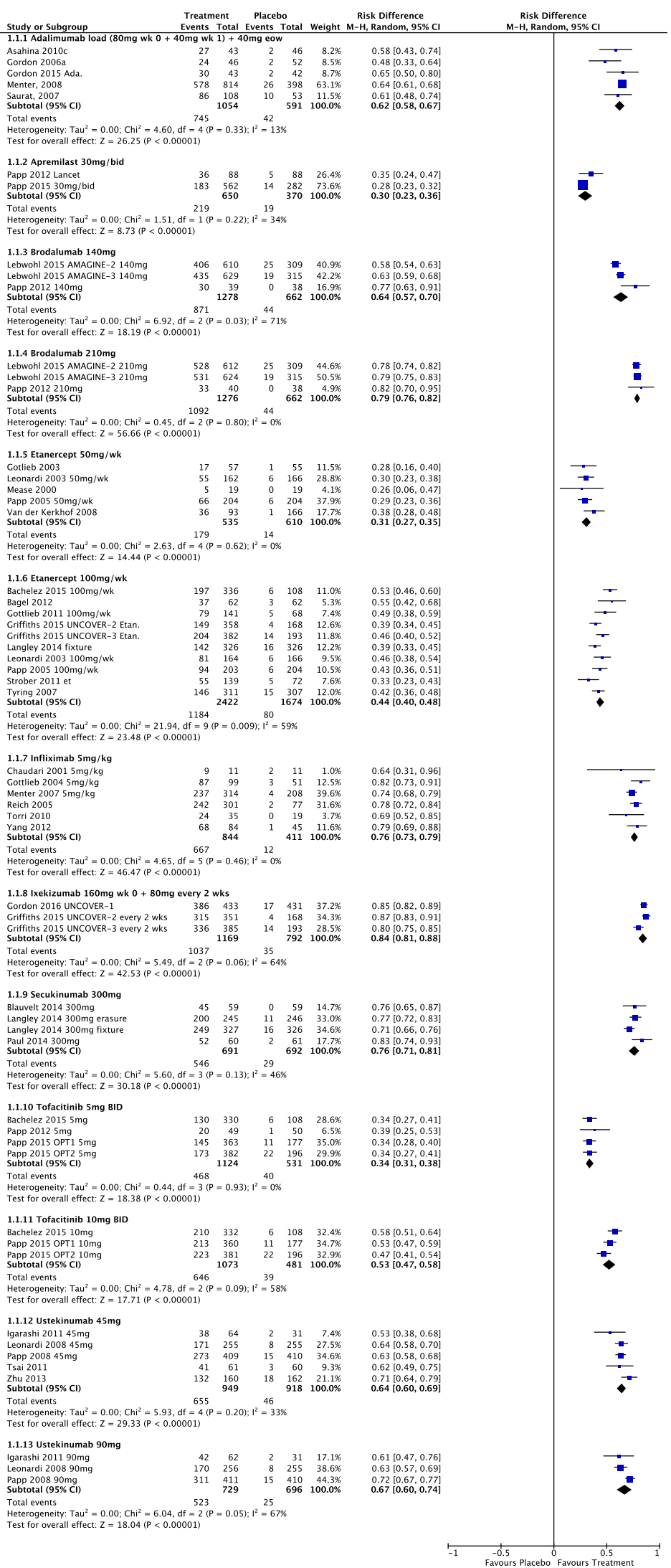


0.58-0.60) [Fig. 3]. When analyzing PASI 75 as an outcome, the estimated NNT for ixekizumab, brodalumab $210 \mathrm{mg}$, infliximab and secukinumab was 1.19, 1.26, 1.31 and 1.31 , respectively. A summary of comparisons is reported in Table 2.

When PASI 90 was used as an outcome, both doses of brodalumab-210 $\mathrm{mg}$ (RD $0.75,95 \%$ CI $0.61-0.89$ ) and $140 \mathrm{mg}$ (RD 0.72, 95\% CI 0.57-0.86-achieved a higher chance of improvement, followed by ixekizumab (RD 0.69, 95\% CI 0.65-0.72). Secukinumab and infliximab showed the same RD, with exactly the same CI (RD $0.53,95 \%$ CI 0.46-0.60). The remaining comparisons are reported in Fig. 4. The overall pooled effect favored treatment in relation to placebo (RD 0.39, 95\% CI 0.38-0.40) [Fig. 5].

Brodalumab $210 \mathrm{mg}$ was also the drug that achieved higher RD if PASI 100 was used as the outcome (RD 0.44, 95\% CI 0.35-0.53). The approved drugs performed as follows: ixekizumab (RD 0.37 , 95\% CI $0.35-0.40$ ), secukinumab (RD $0.28,95 \%$ CI $0.22-0.34$ ), adalimumab (RD 0.18 , 95\% CI 0.12-0.24), and ustekinumab $45 \mathrm{mg}$

\begin{tabular}{|c|c|c|c|c|c|c|c|c|c|c|}
\hline \multirow{2}{*}{$\begin{array}{l}\text { Study or Subgroup } \\
1.1 .1 \text { Treatment }\end{array}$} & \multicolumn{2}{|c|}{ Treatment } & Placebo & $\begin{array}{l}\text { bo } \\
\text { Total }\end{array}$ & \multirow[t]{2}{*}{ Weight } & \multirow[t]{2}{*}{$\begin{array}{c}\text { Risk Difference } \\
\text { M-H, Random, } 95 \% \mathrm{Cl}\end{array}$} & \multicolumn{3}{|c|}{$\begin{array}{c}\text { Risk Difference } \\
\text { M-H, Random, 95\% Cl }\end{array}$} & \\
\hline & & & & & & & & & & \\
\hline $\begin{array}{l}\text { Combined studies } \\
\text { Subtotal }(95 \% \mathrm{Cl})\end{array}$ & 8832 & $\begin{array}{l}13794 \\
13794\end{array}$ & 304 & $\begin{array}{l}6259 \\
6259\end{array}$ & $\begin{array}{l}100.0 \% \\
\mathbf{1 0 0 . 0 \%}\end{array}$ & $\begin{array}{l}0.59[0.58,0.60] \\
0.59[0.58,0.60]\end{array}$ & & & & \\
\hline $\begin{array}{l}\text { Total events } \\
\text { Heterogeneity: Not ap } \\
\text { Test for overall effect }\end{array}$ & $\begin{array}{r}8832 \\
\text { plicable } \\
Z=120\end{array}$ & $58(P<$ & $\begin{array}{r}304 \\
0.00001\end{array}$ & & & & & & & \\
\hline Total $(95 \% \mathrm{Cl})$ & & 13794 & & 6259 & $100.0 \%$ & $0.59[0.58,0.60]$ & & & & 1 \\
\hline Total events & 8832 & & 304 & & & & & & & \\
\hline $\begin{array}{l}\text { Heterogeneity: Not ap } \\
\text { Test for overall effect } \\
\text { Test for subgroup dif }\end{array}$ & $\begin{array}{l}\text { plicable } \\
Z=120 \\
\text { erences: }\end{array}$ & $\begin{array}{l}58(\mathrm{P}< \\
\text { Not app }\end{array}$ & $\begin{array}{l}0.00001 \\
\text { licable }\end{array}$ & & & & $\longmapsto$ & $\begin{array}{cc} & -0.5 \\
\text { Favours Placebo } & 0\end{array}$ & Favours Tre & \\
\hline
\end{tabular}

Fig. 3 Meta-analysis (random-effects model) of the Psoriasis Area and Severity Index $75 \%$ response rate of all treatments combined for moderate to severe psoriasis in randomized, placebo-controlled trials (overall pooled effect). $C I$ confidence interval, $M-H$ Mantel-Haenszel

Table 2 Summary of results for drugs and doses sorted by drug class

\begin{tabular}{|c|c|c|c|c|c|c|c|c|}
\hline \multirow[t]{2}{*}{ Drug class } & \multirow[t]{2}{*}{ Drug/dose } & \multicolumn{2}{|l|}{ PASI 75} & \multicolumn{2}{|l|}{ PASI 90} & \multicolumn{2}{|l|}{ PASI 100} & \multirow{2}{*}{$\begin{array}{l}\text { Primary } \\
\text { endpoin } \\
\text { (weeks) }\end{array}$} \\
\hline & & $\mathrm{RD}(95 \% \mathrm{CI})$ & NNT & $\mathrm{RD}(95 \% \mathrm{CI})$ & NNT & $\mathrm{RD}(95 \% \mathrm{CI})$ & NNT & \\
\hline \multirow[t]{5}{*}{ Anti-TNF } & $\begin{array}{l}\text { Adalimumab load } \\
(80 \mathrm{mg} \text { week } 0+40 \mathrm{mg} \\
\text { week } 1)+40 \mathrm{mg} \text { EOW }\end{array}$ & $0.62(0.58-0.67)$ & 1.61 & $0.43(0.39-0.46)$ & 2.32 & $0.18(0.12-0.24)$ & 5.55 & $12-16$ \\
\hline & Etanercept $100 \mathrm{mg} / \mathrm{wk}$ & $0.44(0.40-0.48)$ & 2.27 & $0.22(0.18-0.25)$ & 4.54 & $0.05(0.04-0.07)$ & 20 & 12 \\
\hline & Etanercept $50 \mathrm{mg} / \mathrm{wk}$ & $0.31(0.27-0.35)$ & 3.22 & $0.10(0.07-0.13)$ & 10 & $0.06(0.01-0.10)$ & 16.6 & 12 \\
\hline & Infliximab $5 \mathrm{mg} / \mathrm{kg}$ & $0.76(0.73-0.79)$ & 1.31 & $0.53(0.46-0.60)$ & 1.88 & ND & ND & 10 \\
\hline & Overall pooled effect & $0.54(0.47-0.60)$ & 1.85 & $0.28(0.21-0.35)$ & 3.57 & $0.10(0.04-0.16)$ & 10 & - \\
\hline \multirow[t]{3}{*}{ Anti-IL-12/23 } & Ustekinumab $90 \mathrm{mg}$ & $0.67(0.60-0.74)$ & 1.49 & $0.42(0.30-0.54)$ & 2.38 & $0.15(0.07-0.22)$ & 6.66 & 12 \\
\hline & Ustekinumab $45 \mathrm{mg}$ & $0.64(0.60-0.69)$ & 1.56 & $0.45(0.35-0.55)$ & 2.22 & $0.16(0.10-0.21)$ & 6.25 & 12 \\
\hline & Overall pooled effect & $0.65(0.62-0.69)$ & 1.53 & $0.44(0.37-0.51)$ & 2.27 & $0.15(0.11-0.19)$ & 6.66 & - \\
\hline \multirow[t]{5}{*}{ Anti-IL-17 } & Brodalumab $210 \mathrm{mg}$ & $0.79(0.76-0.82)$ & 1.26 & $0.75(0.61-0.89)$ & 1.33 & $0.44(0.35-0.53)$ & 2.27 & 12 \\
\hline & Brodalumab $140 \mathrm{mg}$ & $0.64(0.57-0.70)$ & 1.56 & $0.72(0.57-0.86)$ & 1.38 & $0.26(0.23-0.30)$ & 3.84 & 12 \\
\hline & $\begin{array}{l}\text { Ixekizumab } \\
160 \mathrm{mg} \text { week } 0 \\
\text { and } 80 \mathrm{mg} \text { every } 2 \text { weeks }\end{array}$ & $0.84(0.81-0.88)$ & 1.19 & $0.69(0.65-0.72)$ & 1.44 & $0.37(0.35-0.40)$ & 2.70 & 12 \\
\hline & Secukinumab $300 \mathrm{mg}$ & $0.76(0.71-0.81)$ & 1.31 & $0.53(0.46-0.60)$ & 1.88 & $0.28(0.22-0.34)$ & 3.57 & 12 \\
\hline & Overall pooled effect & $0.76(0.70-0.82)$ & 1.31 & $0.61(0.54-0.68)$ & 1.63 & $0.35(0.30-0.40)$ & 2.85 & - \\
\hline \multirow{4}{*}{$\begin{array}{l}\text { Small molecule } \\
\text { inhibitors } \\
\text { (anti-JAK/anti-PD4) }\end{array}$} & Tofacitinib $10 \mathrm{mg}$ & $0.53(0.47-0.58)$ & 1.88 & $0.36(0.33-0.39)$ & 2.77 & ND & ND & 12 \\
\hline & Tofacitinib $5 \mathrm{mg}$ & $0.34(0.31-0.38)$ & 2.94 & $0.19(0.17-0.22)$ & 5.26 & ND & ND & 12 \\
\hline & Apremilast $30 \mathrm{mg}$ bid & $0.30(0.23-0.36)$ & 3.33 & ND & ND & ND & ND & 16 \\
\hline & Overall pooled effect & $0.43(0.30-0.55)$ & 2.32 & $0.27(0.13-0.42)$ & 3.7 & ND & ND & - \\
\hline
\end{tabular}

$P A S I$ Psoriasis Area and Severity Index, $R D$ risk difference, $C I$ confidence interval, $N N T$ number needed to treat, $E O W$ every other week, bid twice daily, JAK Janus kinase, $P D 4$ phosphodiesterase $4, N D$ not determined, $T N F$ tumor necrosis factor, $I L$ interleukin 
Fig. 4 Meta-analysis (randomeffects model) of the Psoriasis Area and Severity Index 90\% response rate of biologic and small molecule inhibitor therapies for moderate to severe psoriasis in randomized, placebo-controlled trials. $C I$ confidence interval, $M-H$ Mantel-Haenszel, EOW every other week, $d f$ degrees of freedom, bid twice daily

\begin{tabular}{|c|c|c|c|c|}
\hline Study or Subgroup & $\begin{array}{c}\text { Treatm } \\
\text { Events }\end{array}$ & $\begin{array}{l}\text { nent } \\
\text { Total } \\
\end{array}$ & $\begin{array}{l}\text { Placebo } \\
\text { Events T }\end{array}$ & Total \\
\hline 1.1.3 Adalimumab load $(80 \mathrm{mg}$ wk $0+$ & $40 \mathrm{mg} \mathrm{wk}$ & k1) +40 & Omg eow & \\
\hline Gordon 2015 Ada. & 19 & 43 & 1 & 42 \\
\hline $\begin{array}{l}\text { Menter, } 2008 \\
\text { Subtotal (95\% CI) }\end{array}$ & 366 & $\begin{array}{l}814 \\
857\end{array}$ & 8 & 398 \\
\hline Total events & 385 & & 9 & \\
\hline $\begin{array}{l}\text { Heterogeneity: } \mathrm{Tau}^{2}=0.00 ; \mathrm{Chi}^{2}=0.02 \\
\text { Test for overall effect: } Z=23.45 \text { ( } \mathrm{P}<0 \text {. }\end{array}$ & $\begin{array}{l}\mathrm{df}=1(\mathrm{P} \\
0001)\end{array}$ & $=0.89$ & $: 1^{2}=0 \%$ & \\
\hline 1.1 .7 Brodalumab $140 \mathrm{mg}$ & & & & \\
\hline $\begin{array}{l}\text { Papp } 2012140 \mathrm{mg} \\
\text { Subtotal }(95 \% \mathrm{Cl})\end{array}$ & 28 & $\begin{array}{l}39 \\
39\end{array}$ & 0 & $\begin{array}{l}38 \\
\mathbf{3 8}\end{array}$ \\
\hline Total events & 28 & & 0 & \\
\hline $\begin{array}{l}\text { Heterogeneity: Not applicable } \\
\text { Test for overall effect: } Z=9.73 \text { ( } P<0.0\end{array}$ & 001) & & & \\
\hline 1.1.8 Brodalumab $210 \mathrm{mg}$ & & & & \\
\hline Papp $2012210 \mathrm{mg}$ & 30 & 40 & 0 & 38 \\
\hline Subtotal $(95 \% \mathrm{Cl})$ & & 40 & & 38 \\
\hline Total events & 30 & & 0 & \\
\hline Heterogeneity: Not applicable & & & & \\
\hline Test for overall effect: $Z=10.64$ ( $P<0$. & 0001) & & & \\
\hline 1.1.13 Etanercept $50 \mathrm{mg} / \mathrm{wk}$ & & & & \\
\hline Leonardi $200350 \mathrm{mg} / \mathrm{wk}$ & 19 & 162 & 1 & 166 \\
\hline Papp $200550 \mathrm{mg} / \mathrm{wk}$ & 20 & 204 & 1 & 204 \\
\hline Strober 2011 & 19 & 139 & 3 & 72 \\
\hline Van der Kerkhof 2008 & 13 & & 1 & \\
\hline Subtotal $(95 \% \mathrm{Cl})$ & 13 & 601 & 1 & 488 \\
\hline Total events & 71 & & 6 & \\
\hline $\begin{array}{l}\text { Heterogeneity: } \mathrm{Tau}^{2}=0.00 ; \mathrm{Chi}^{2}=0.41 \\
\text { Test for overall effect: } Z=7.14(\mathrm{P}<0.0\end{array}$ & $\begin{array}{l}d f=3(P \\
001) \\
0.1\end{array}$ & $=0.94)$ & $: 1^{2}=0 \%$ & \\
\hline 1.1.14 Etanercept $100 \mathrm{mg} / \mathrm{wk}$ & & & & \\
\hline $\begin{array}{l}\text { Bachelez } 2015100 \mathrm{mg} / \text { week } \\
\text { Bek }\end{array}$ & 108 & 336 & 1 & 108 \\
\hline Bagel 2012 & 16 & 62 & 1 & 62 \\
\hline Griffiths UNCOVER-2 2015 Etan. & 67 & 358 & 1 & 168 \\
\hline Griffiths UNCOVER-3 2015 Etan. & 98 & 382 & 6 & 193 \\
\hline Langley 2014 fixture & 67 & 326 & 5 & 326 \\
\hline Leonardi $2003100 \mathrm{mg} / \mathrm{wk}$ & 36 & 164 & 1 & 166 \\
\hline Papp $2005100 \mathrm{mg} / \mathrm{wk}$ & 39 & 203 & 1 & 204 \\
\hline Tyring 2007 & 65 & & 3 & \\
\hline Subtotal $(95 \% \mathrm{Cl})$ & 63 & 2142 & 1 & 1534 \\
\hline Total events & 496 & & 19 & \\
\hline $\begin{array}{l}\text { Heterogeneity: } \mathrm{Tau}^{2}=0.00 ; \mathrm{Ch}^{2}=18.2 \\
\text { Test for overall effect: } Z=13.67(\mathrm{P}<0 .\end{array}$ & $\begin{array}{l}d f=7(P) \\
0001)\end{array}$ & $(P=0.01$ & 1); $I^{2}=62$ & \\
\hline 1.1.25 Infliximab $5 \mathrm{mg}$ & & & & \\
\hline Gottlieb $20045 \mathrm{mg} / \mathrm{kg}$ & 57 & 99 & 1 & 51 \\
\hline Menter $20075 \mathrm{mg} / \mathrm{kg}$ & 142 & 314 & 1 & 208 \\
\hline Reich 2005 & 172 & 301 & 1 & 77 \\
\hline Yang 2012 & 48 & 84 & 0 & 45 \\
\hline Subtotal $(95 \% \mathrm{Cl})$ & 40 & 798 & 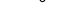 & 381 \\
\hline Total events & 419 & & 3 & \\
\hline $\begin{array}{l}\text { Heterogeneity: } \mathrm{Tau}^{2}=0.00: \mathrm{Ch}^{2}=9.38 \\
\text { Test for overall effect: } Z=14.95(\mathrm{P}<0 .\end{array}$ & $\begin{array}{l}d f=3(P \\
0001) \\
0.01\end{array}$ & $P=0.02)$ & $1^{2}=68 \%$ & \\
\hline 1.1 .31 Ixekizumab $160 \mathrm{mg}$ wk $0+80 \mathrm{~m}$ & every $2 \mathrm{v}$ & wks & & \\
\hline Gordon 2016 UNCOVER-1 & 307 & 433 & 2 & 431 \\
\hline Griffiths 2015 UNCOVER- 2 every 2 wks & 248 & 351 & 1 & 168 \\
\hline Griffiths 2015 UNCOVER-3 every 2 wks & 262 & 385 & 6 & 193 \\
\hline Subtotal $(95 \% \mathrm{Cl})$ & & 1169 & & 792 \\
\hline Total events & 817 & & 9 & \\
\hline $\begin{array}{l}\text { Heterogeneity: } \operatorname{Tau}^{2}=0.00 ; \mathrm{Chi}^{2}=2.87 \\
\text { Test for overall effect: } Z=40.53(\mathrm{P}<0 .\end{array}$ & $\begin{array}{l}\mathrm{df}=2(\mathrm{P} \\
0001)\end{array}$ & $P=0.24$ & $; I^{2}=30 \%$ & \\
\hline 1.1 .35 Secukinumab $300 \mathrm{mg}$ & & & & \\
\hline Blauvelt $2014300 \mathrm{mg}$ & 36 & 59 & 0 & 59 \\
\hline Langley $2014300 \mathrm{mg}$ erasure & 145 & 245 & 3 & 248 \\
\hline $\begin{array}{l}\text { Langley } 2014300 \mathrm{mg} \text { fixture } \\
\text { Lits }\end{array}$ & 175 & 327 & 5 & 326 \\
\hline Paul $2014300 \mathrm{mg}$ & 24 & 60 & 0 & 61 \\
\hline Subtotal $(95 \% \mathrm{Cl})$ & & 691 & & 694 \\
\hline Total events & 380 & & 8 & \\
\hline $\begin{array}{l}\text { Heterogeneity: } \operatorname{Tau}^{2}=0.00: \mathrm{Ch}^{2}=8.04 \\
\text { Test for overall effect: } Z=15.17(\mathrm{P}<0 .\end{array}$ & $\begin{array}{l}d f=3(P \\
0001) \\
0.0\end{array}$ & $=0.05$ & $1^{2}=63 \%$ & \\
\hline 11.36 Tofacitinib $5 \mathrm{mg}$ BID & & & & \\
\hline $\begin{array}{l}\text { 1.1.36 Tofacitinib } 5 \mathrm{mg} \mathrm{BID} \\
\text { Bachelez } 20155 \mathrm{mg}\end{array}$ & & & & \\
\hline $\begin{array}{l}\text { Bachelez } 20155 \mathrm{mg} \\
\text { Papp } 2015 \text { OPT1 5mg }\end{array}$ & 69 & 330 & 1 & 108 \\
\hline $\begin{array}{l}\text { Papp } 2015 \text { OPT1 } 5 \mathrm{mg} \\
\text { Papp } 2015 \text { OPT2 5mg }\end{array}$ & 72 & 363 & 1 & 177 \\
\hline $\begin{array}{l}\text { Papp } 2015 \text { OPT2 } 5 \mathrm{mg} \\
\text { Subtotal }(95 \% \mathrm{Cl})\end{array}$ & 93 & 382 & 10 & 196 \\
\hline Total events & 234 & 1075 & 12 & 481 \\
\hline Heterogeneity: $\mathrm{Tau}^{2}=0.00 ; \mathrm{Chi}^{2}=0.06$ & $d f=2(P$ & $P=0.97)$ & $; 1^{2}=0 \%$ & \\
\hline Test for overall effect: $Z=14.07(P<0$. & $0001)$ & & & \\
\hline 1.1.37 Tofacitinib $10 \mathrm{mg}$ BID & & & & \\
\hline Bachelez $201510 \mathrm{mg}$ & 119 & 332 & 1 & 108 \\
\hline Papp 2015 OPT1 $10 \mathrm{mg}$ & 140 & 360 & 1 & 177 \\
\hline & 148 & & 10 & \\
\hline $\begin{array}{l}\text { Pubtotal }(95 \% \mathrm{Cl}) \\
\text { Sumg }\end{array}$ & 148 & $\begin{array}{r}381 \\
1073\end{array}$ & 10 & 481 \\
\hline Total events & 407 & & 12 & \\
\hline $\begin{array}{l}\text { Heterogeneity: } \mathrm{Tau}^{2}=0.00 ; \mathrm{Chi}^{2}=1.52 \\
\text { Test for overall effect: } Z=22.32(\mathrm{P}<0 .\end{array}$ & $d f=2(P=$ & $=0.47)$ & $: I^{2}=0 \%$ & \\
\hline Test for overall effect: $Z=22.32(P<0$. & 2001) & & & \\
\hline 1.1.39 Ustekinumab $45 \mathrm{mg}$ & & & & \\
\hline Igarashi $201145 \mathrm{mg}$ & 21 & 64 & 1 & 31 \\
\hline Leonardi $200845 \mathrm{mg}$ & 106 & 255 & 5 & 255 \\
\hline Papp $200845 \mathrm{mg}$ & 173 & 409 & 3 & 410 \\
\hline Tsai 2011 & 30 & 61 & 1 & 60 \\
\hline Zhu 2013 & 107 & 160 & 5 & 162 \\
\hline Subtotal $(95 \% \mathrm{Cl})$ & & 949 & & 918 \\
\hline & 437 & & 15 & \\
\hline Heterogeneity: $\mathrm{Tau}^{2}=0.01 ; \mathrm{Chi}^{2}=32.6$ & $\mathrm{df}=4(\mathrm{P}$ & $(P<0.00$ & $0001) ; 1^{2}$ & $=88 \%$ \\
\hline Test for overall effect: $Z=8.68(P<0.0$ & 01) & & & \\
\hline 1.1 .40 Ustekinumab $90 \mathrm{mg}$ & & & & \\
\hline Igarashi $201190 \mathrm{mg}$ & 27 & 62 & 1 & 31 \\
\hline Leonardi $200890 \mathrm{mg}$ & 94 & 256 & 5 & 255 \\
\hline Papp $200890 \mathrm{mg}$ & 209 & 411 & 3 & 410 \\
\hline Subtotal $(95 \% \mathrm{Cl})$ & & 729 & & 696 \\
\hline Total events & 330 & & 9 & \\
\hline Heterogeneity: $\mathrm{Tau}^{2}=0.01 ; \mathrm{Chi}^{2}=14.9$ & $\mathrm{df}=2(\mathrm{P}$ & $(P=0.00$ & 006); $1^{2}=$ & $=87 \%$ \\
\hline Test for overall effect: $Z=7.15(P<0.0$ & & & & \\
\hline & & & & \\
\hline
\end{tabular}




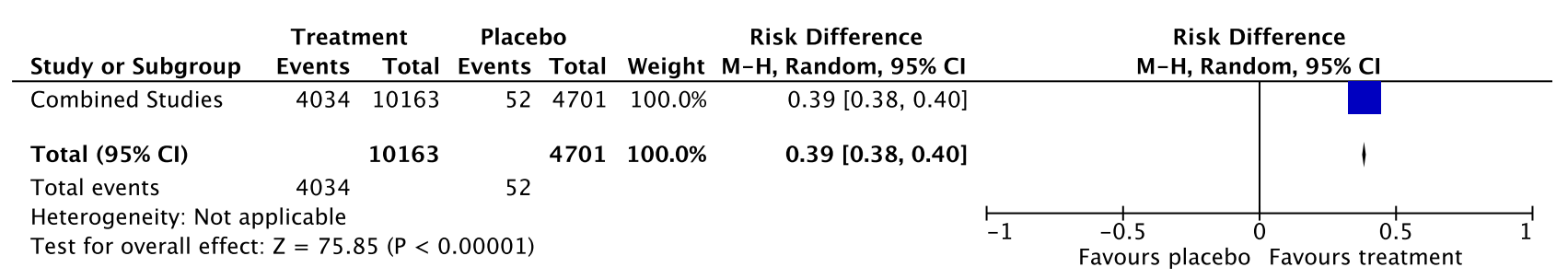

Fig. 5 Meta-analysis (random-effects model) of the Psoriasis Area and Severity Index 90\% response rate of all treatments combined for moderate to severe psoriasis in randomized, placebo-controlled trials (overall pooled effect). $C I$ confidence interval, $M$ - $H$ Mantel-Haenszel

(RD 0.16 , 95\% CI 0.10-0.21). Other comparisons are reported in Fig. 6. The overall pooled effect of treatment versus placebo was also favorable towards treatment (RD 0.24, 95\% CI 0.23-0.25) [Fig. 7].

Heterogeneity $\left(I^{2}\right)$ on the PASI 75 outcome analysis was below $40 \%$ in the following drugs: adalimumab $\left(I^{2}=13 \%\right)$, apremilast $\left(I^{2}=34 \%\right)$, brodalumab $210 \mathrm{mg}$ $\left(I^{2}=0 \%\right)$, etanercept $50 \mathrm{mg} \quad\left(I^{2}=0 \%\right)$, infliximab $\left(I^{2}=0 \%\right)$, tofacitinib $5 \mathrm{mg}\left(I^{2}=0 \%\right)$, and ustekinumab $45 \mathrm{mg}\left(I^{2}=33 \%\right)$. Moderate heterogeneity was found in the etanercept $100 \mathrm{mg} \quad\left(I^{2}=59 \%\right)$, secukinumab $\left(I^{2}=46 \%\right)$, and tofacitinib $10 \mathrm{mg}\left(I^{2}=58 \%\right)$ groups. In addition, substantial heterogeneity was seen in the following groups: brodalumab $140 \mathrm{mg}\left(\mathrm{I}^{2}=71 \%\right)$, ixekizumab $\left(I^{2}=64 \%\right)$, and ustekinumab $90 \mathrm{mg}\left(I^{2}=67 \%\right)$ [Fig. 2]. Heterogeneity for remaining outcomes (PASI 90 and 100) have been reported in Figs. 4 and 6.

The funnel plot of the PASI 75 outcome did not disclose discrepancies with regard to the magnitude of the effect measured and study size. PASI 90 and PASI 100 funnel plots are not typical, and the lack of symmetry observed may be an indication of publication bias (Online Resource 3,4 , and 5).

Meta-regression was performed to evaluate the contribution of mean baseline PASI, previous use of biologics, and duration of the disease to the heterogeneity among studies (Online Resource 6). None of these variables explained the heterogeneity.

\section{Discussion}

In the present review, anti-IL-17 drugs performed very well. Ixekizumab presented the higher RD in the primary outcome (PASI 75), while brodalumab (210 mg) performed well, following ixekizumab on the primary outcome and achieving a higher RD on both secondary outcomes (PASI 90 and PASI 100). Nevertheless, as the CI overlapped, ixekizumab, brodalumab $210 \mathrm{mg}$, and secukinumab should be considered as having similar performances, even with different RDs. On the other hand, ixekizumab and brodalumab $210 \mathrm{mg}$ were superior to all remaining drugs, with the exception of infliximab and secukinumab, when PASI 75 was the outcome (no overlapping of the $\mathrm{CI}$ ).

When analyzing PASI 90 as an outcome, brodalumab (both doses) had the higher RD, but the performance of ixekizumab was similar to the overlapped CI. Nevertheless, it is important to emphasize that only one brodalumab study [11] (both dosages) could be identified that used PASI 90 as an outcome; however, a low number of patients were enrolled in this study. Therefore, the results regarding brodalumab at this particular outcome should be considered with caution. Ixekizumab was also the best performing drug, and was superior than all the remaining approved medications (no overlapping of the CI). Ixekizumab also showed the highest RD among approved drugs at PASI 100, and was superior to all remaining medications (no overlapping of the CI) when drugs were compared with placebo. At the same outcome, brodalumab $210 \mathrm{mg}$ achieved a higher RD than ixekizumab, but effectiveness was comparable as the CI overlapped. The number of studies included in the analysis of PASI 100 outcome for brodalumab was higher, enrolling more than 1200 patients, which makes these findings more robust than the findings for brodalumab when PASI 90 was analyzed.

Among the newer small molecule inhibitor drugs, tofacitinib, an anti-Janus kinase 1, also performed well at a dose of $10 \mathrm{mg}$, being superior to lower-dose etanercept when compared with placebo, and comparable to higherdose etanercept and adalimumab and low-dose brodalumab (overlapping CI), considering PASI 75 as the primary outcome.

On the other hand, apremilast, an anti-phosphodiesterase 4 drug, performed poorly. Nevertheless, it is comparable to low-dose etanercept (50 mg/week) and low-dose tofacitinib $(5 \mathrm{mg})$ at the primary outcome. PASI 90 and 100 analyses could not be performed for apremilast as no studies that could supply the appropriate data were identified.

In accordance with previous meta-analyses [16, 52], infliximab also performed well among approved biologics. At both dosages (45 and $90 \mathrm{mg}$ ), ustekinumab had basically the same performance, which may be explained by the fact that the included studies did not stratify the analysis by patient weight and dosage. The order of 


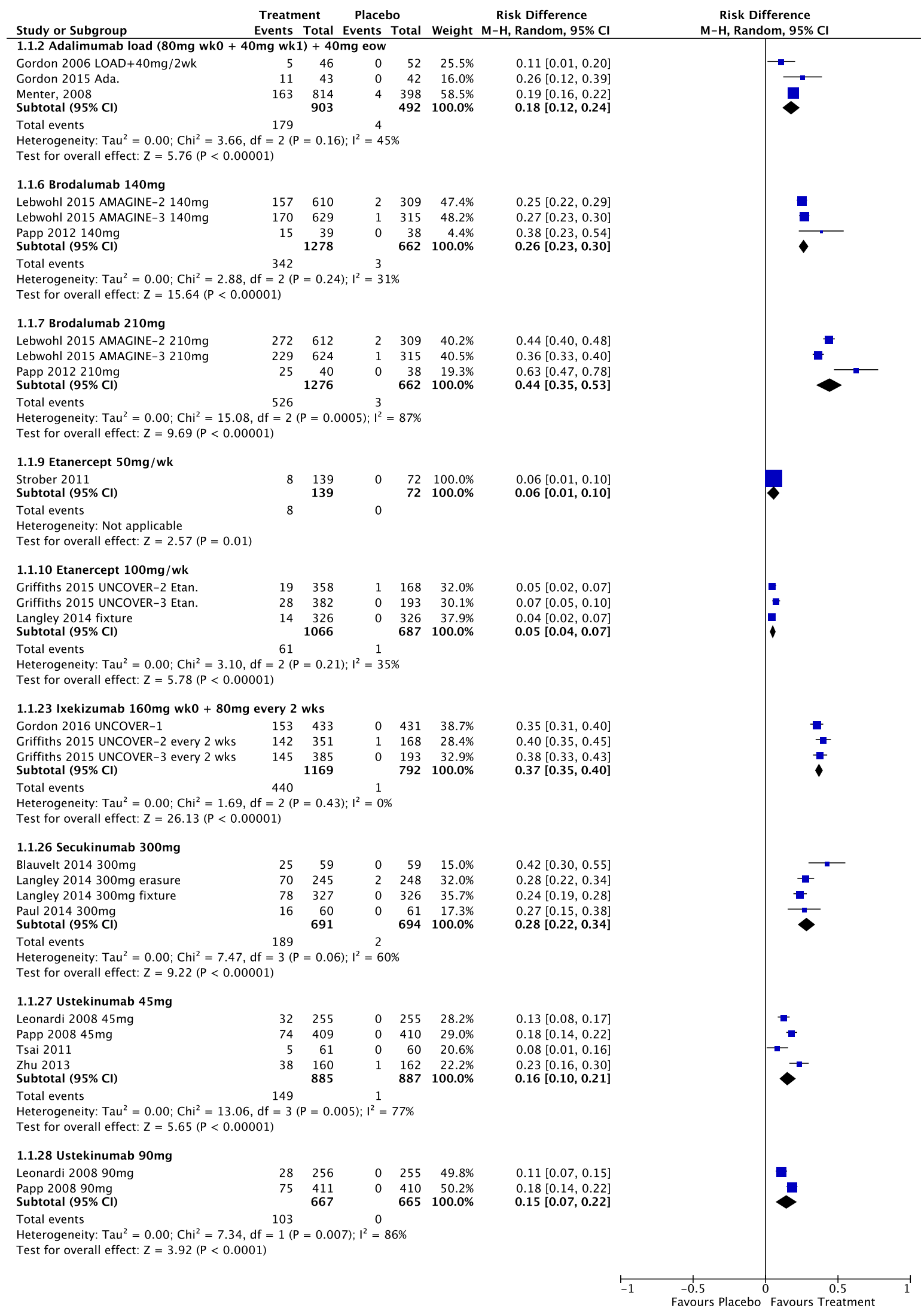


4Fig. 6 Meta-analysis (random-effects model) of the Psoriasis Area and Severity Index $100 \%$ response rate of biologic and small molecule inhibitor therapies for moderate to severe psoriasis in randomized, placebo-controlled trials. $C I$ confidence interval, $M-H$ Mantel-Haenszel, EOW every other week, $d f$ degrees of freedom

effectiveness, measured by RD, in which infliximab $5 \mathrm{mg} /$ $\mathrm{kg}$, ustekinumab $90 \mathrm{mg}$, ustekinumab $45 \mathrm{mg}$, adalimumab, etanercept $100 \mathrm{mg}$, and etanercept $50 \mathrm{mg}$ were positioned in this meta-analysis, was the same as that reported by Schmitt et al. in a recent meta-analysis [16]. Using a different meta-analysis methodology (Bayesian, network meta-analysis), Reich et al. [54] identified a similar ranking with regard to the chance of a PASI 75 response, with the exception of the etanercept $100 \mathrm{mg}$ response rate, which was not contemplated in the study. It is important to note that a rank of RD is somewhat deceiving as the CI may overlap. Nevertheless, the concordance of the rank seen in the work of Reich et al., which used a Bayesian analysis, and the RD rank found in this work, indicates a consistent trend [54].

It is important to emphasize that the objective of this meta-analysis was to compare active treatments against placebo. A limitation to this work is that it is not inherently designed to make indirect comparisons of active treatments and, as previously stated, overlapping CIs determine that drugs are equally effective. A further Bayesian network meta-analysis should be performed to address this issue and, eventually, allow indirect comparisons to rank active treatments.

Considering approved doses and PASI 75 as an outcome, heterogeneity inside each group has been found to be low $\left(I^{2} \leq 40 \%\right)$ or moderate $\left(I^{2}>40, \leq 60 \%\right)$ [20] for all comparisons, except for ustekinumab $90 \mathrm{mg}\left(I^{2}=67 \%\right)$, brodalumab $\left(I^{2}=71 \%\right)$, and ixekizumab $\left(I^{2}=64 \%\right)$. The heterogeneity of ustekinumab at a higher dose may be explained by the grouped analysis of populations of different weights $(>100$ or $<100 \mathrm{~kg}$ ). After performing sensitivity analysis, the heterogeneity found in the brodalumab subgroup was found to be due to a phase II study [11] with a low number of participants; heterogeneity for the brodalumab subgroup decreased to $48 \%$ after exclusion of this particular study. No reason was found to account for heterogeneity in the ixekizumab group, neither in metaregression (Online Resource 6) nor in the sensitivity analysis. Heterogeneity of the pooled overall efficacy of active treatments compared with placebo was high, but this intergroup heterogeneity was expected due to the number of different treatments analyzed and the unit of analysis error. Meta-regression was performed to analyze the impact of PASI score prior to treatment, duration of psoriasis, or previous use of biologic or small molecule inhibitor drugs on heterogeneity. None of the pre-defined variables influenced the results. Random-effects metaanalysis was performed as an attempt to incorporate heterogeneity.

Risk of bias assessment showed a small percentage of high risk of bias categorization among the included studies. On the other hand, $50 \%$ of the studies did not explicitly disclose the random sequence generation or allocation concealment (selection bias) well enough, although being categorized as having an unclear risk of bias.

The funnel plot of the primary outcome (PASI 75) was interpreted as being symmetrical and therefore it is less likely that publication bias may have been present. Larger studies are based on the mean RD at the top of the plot. The lack of smaller studies is responsible for the empty space found at the bottom of the plot, but one can assume this is due to the inclusion and exclusion criteria of the systematic review (only placebo-controlled RCTs). Two smaller studies are found on the plot but they are symmetrically positioned on each side of the mean RD. PASI 90 and 100 funnel plot were asymmetric, and both showed smaller studies to be highly efficacious, resulting in a plot that had an empty lower left quadrant. One explanation for this asymmetry is that studies that evaluated less effective drugs may have chosen not to report PASI 90 and 100, while studies evaluating more effective drugs tend to report these outcomes. Therefore, publication bias cannot be ruled out.

The time of outcomes assessment may also be a limitation in the interpretation of the results. As the majority of RCTs used a 12-week timeframe, extrapolation of the results for longer periods may not be appropriate.

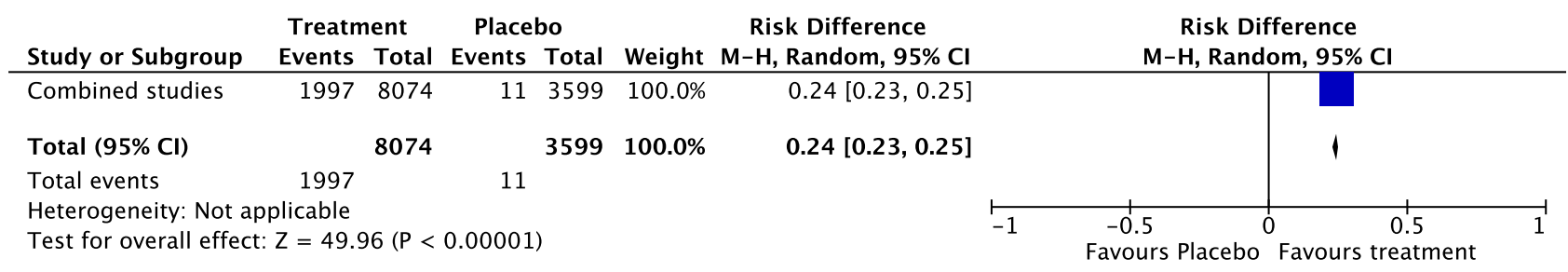

Fig. 7 Meta-analysis (random-effects model) of the Psoriasis Area and Severity Index 100\% response rate of all treatments combined for moderate to severe psoriasis in randomized, placebo-controlled trials (overall pooled effect). $C I$ confidence interval, $M-H$ Mantel-Haenszel 


\section{Conclusions}

This meta-analysis showed that biologics and small molecule inhibitors are highly effective for the treatment of moderate to severe psoriasis, and that anti-IL-17 drugs have the same, or even greater, efficacy than anti-tumor necrosis factor (TNF) and anti-IL-12/23 drugs when PASI 75 or PASI 90 are used as the outcome. If PASI 100 is used as the outcome, newer drugs such as anti-IL-17 tend to perform better than anti-TNF and anti-IL-12/23 drugs. As the number of newer biologic and small molecule inhibitor drugs increases, the efficacy of these drugs compared with placebo, found in this meta-analysis, can help doctors to choose what the most appropriate treatment is for each particular patient.

\section{Compliances with Ethical Standards}

\section{Funding None.}

Conflict of interest André Vicente Esteves de Carvalho has received research support and is a speaker/advisory board program participant receiving honoraria for Abvie, Jansen, Novartis and Leo Pharma. Rodrigo Pereira Duquia, Bernardo Lessa Horta and Renan Rangel Bonamigo have no conflicts of interest.

Open Access This article is distributed under the terms of the Creative Commons Attribution-NonCommercial 4.0 International License (http://creativecommons.org/licenses/by-nc/4.0/), which permits any noncommercial use, distribution, and reproduction in any medium, provided you give appropriate credit to the original author(s) and the source, provide a link to the Creative Commons license, and indicate if changes were made.

\section{References}

1. Durham LE, Kirkham BW, Taams LS. Contribution of the IL-17 pathway to psoriasis and psoriatic arthritis. Curr Rheumatol Rep. 2015;17(8):55.

2. Menter A, Gottlieb A, Feldman SR, et al. Guidelines of care for the management of psoriasis and psoriatic arthritis. J Am Acad Dermatol. 2008;58(5):826-50.

3. Gelfand JM, Neimann AL, Shin DB, et al. Risk of myocardial infarction in patients with psoriasis. JAMA. 2006;296(14): 1735-41.

4. Chaudhari U, Romano P, Mulcahy LD, et al. Efficacy and safety of infliximab monotherapy for plaque-type psoriasis: a randomised trial. Lancet. 2001;357(9271):1842-7.

5. Gordon KB, Langley RG, Leonardi C, et al. Clinical response to adalimumab treatment in patients with moderate to severe psoriasis: double-blind, randomized controlled trial and open-label extension study. J Am Acad Dermatol. 2006;55(4):598-606.

6. Krueger GG, Langley RG, Leonardi C, et al. A human interleukin-12/23 monoclonal antibody for the treatment of psoriasis. N Engl J Med. 2007;356(6):580-92.

7. Papp K, Cather JC, Rosoph L, et al. Efficacy of apremilast in the treatment of moderate to severe psoriasis: a randomised controlled trial. Lancet. 2012;380(9843):738-46.
8. Mease PJ, Goffe BS, Metz J, et al. Etanercept in the treatment of psoriatic arthritis and psoriasis: a randomised trial. Lancet. 2000;356(9227):385-90.

9. Leonardi C, Matheson R, Zachariae C, et al. Anti-interleukin-17 monoclonal antibody ixekizumab in chronic plaque psoriasis. N Engl J Med. 2012;366(13):1190-9.

10. Rich P, Sigurgeirsson B, Thaci DP, et al. Secukinumab induction and maintenance therapy in moderate-to-severe plaque psoriasis: a randomised, double-blind, placebo-controlled, phase II regimen-finding study. Br J Dermatol. 2013;168:402-11.

11. Papp KA, Leonardi C, Menter A, et al. Brodalumab, an antiinterleukin-17-receptor antibody for psoriasis. N Engl J Med. 2012;366(13):1181-9.

12. Sofen H, Li K, Smith S, et al. Guselkumab (an IL-23-specific $\mathrm{mAb}$ ) demonstrates clinical and molecular response in patients with moderate-to-severe psoriasis. J Allergy Clin Immunol. 2014;133(4):1032-40.

13. Reich K, Ortonne JP, Gottlieb AB, et al. Successful treatment of moderate to severe plaque psoriasis with the PEGylated $\mathrm{Fab}^{\prime}$ certolizumab pegol: results of a phase II randomized, placebocontrolled trial with a re-treatment extension. Br J Dermatol. 2012;167(1):180-90.

14. Papp KA, Menter A, Strober B, et al. Efficacy and safety of tofacitinib, an oral Janus kinase inhibitor, in the treatment of psoriasis: a phase $2 \mathrm{~b}$ randomized placebo-controlled dose-ranging study. Br J Dermatol. 2012;167(3):668-77.

15. Gordon KB, Langley RG, Gottlieb AB, et al. A phase iii, randomized, controlled trial of the fully human IL-12/23 mAb briakinumab in moderate-to-severe psoriasis. J Invest Dermatol. 2011;132(2):304-14.

16. Schmitt J, Rosumeck S, Thomaschewski G, et al. Efficacy and safety of systemic treatments for moderate-to-severe psoriasis: meta-analysis of randomized controlled trials. Br J Dermatol. 2014;170(2):274-303.

17. Xiong H-Z, Gu J-Y, He Z-G, et al. Efficacy and safety of secukinumab in the treatment of moderate to severe plaque psoriasis: a meta-analysis of randomized controlled trials. Int $\mathbf{J}$ Clin Exp Med. 2015;8(3):3156-72.

18. Chen Y, Qian T, Zhang D, et al. Clinical efficacy and safety of anti-IL-17 agents for the treatment of patients with psoriasis. Immunotherapy. 2015;7(9):1023-37.

19. Liberati A, Altman DG, Tetzlaff J, et al. The PRISMA statement for reporting systematic reviews and meta-analyses of studies that evaluate health care interventions: explanation and elaboration. PLoS Med. 2009;6(7):e1000100-28.

20. Higgins JPT, Green S, editors. Cochrane handbook for systematic reviews of interventions. Version 5.1.0 [updated March 2011]. The Cochrane Collaboration, 2011. Available at: http://www. cochrane-handbook.org. Accessed 21 July 2016

21. Lebwohl M, Strober B, Menter A, et al. Phase 3 studies comparing brodalumab with ustekinumab in psoriasis. N Engl J Med. 2015;373(14):1318-28.

22. Asahina A, Nakagawa H, Etoh T, et al. Adalimumab in Japanese patients with moderate to severe chronic plaque psoriasis: efficacy and safety results from a phase II/III randomized controlled study. J Dermatol. 2010;37(4):299-310.

23. Bagel J, Lynde C, Tyring S. Moderate to severe plaque psoriasis with scalp involvement: a randomized, double-blind, placebocontrolled study of etanercept. J Am Acad Dermatol. 2012;67(1):86-92.

24. Blauvelt A, Prinz JC, Gottlieb AB, et al. Secukinumab administration by pre-filled syringe: efficacy, safety and usability results from a randomized controlled trial in psoriasis (FEATURE). Br J Dermatol. 2015;172(2):484-93.

25. Gottlieb A, Menter A, Mendelsohn A, et al. Ustekinumab, a human interleukin 12/23 monoclonal antibody, for psoriatic 
arthritis: randomised, double-blind, placebo-controlled, crossover trial. Lancet. 2009;373(9664):633-40.

26. Gottlieb AB, Matheson RT, Lowe N, et al. A randomized trial of etanercept as monotherapy for psoriasis. Arch Dermatol. 2003;139(12):1627-32.

27. Igarashi A, Kato T, Kato M, et al. Efficacy and safety of ustekinumab in Japanese patients with moderate-to-severe plaque-type psoriasis: long-term results from a phase $2 / 3$ clinical trial. J Dermatol. 2011;39(3):242-52.

28. Van De Kerkhof P, Segaert S, Lahfa M, et al. Once weekly administration of etanercept $50 \mathrm{mg}$ is efficacious and well tolerated in patients with moderate-to-severe plaque psoriasis: a randomized controlled trial with open-label extension. $\mathrm{Br} \mathbf{J}$ Dermatol. 2008;159(5):1177-85.

29. Langley RG, Elewski BE, Lebwohl M, et al. Secukinumab in plaque psoriasis: results of two phase 3 trials. N Engl J Med. 2014;371(4):326-38.

30. Leonardi CL, Kimball AB, Papp KA, et al. Efficacy and safety of ustekinumab, a human interleukin-12/23 monoclonal antibody, in patients with psoriasis: 76-week results from a randomised, double-blind, placebo-controlled trial (PHOENIX 1). Lancet. 2008;371(9625):1665-74.

31. Leonardi CL, Powers JL, Matheson RT, et al. Etanercept as monotherapy in patients with psoriasis. $\mathrm{N}$ Engl $\mathrm{J}$ Med. 2003;349(21):2014-22.

32. Menter A, Tyring SK, Gordon K, et al. Adalimumab therapy for moderate to severe psoriasis: a randomized, controlled phase III trial. J Am Acad Dermatol. 2008;58(1):106-15.

33. Menter A, Feldman SR, Weinstein GD, et al. A randomized comparison of continuous vs. intermittent infliximab maintenance regimens over 1 year in the treatment of moderate-to-severe plaque psoriasis. J Am Acad Dermatol. 2007;56(1):31.e1-15.

34. Papp KA, Langley RG, Sigurgeirsson B, et al. Efficacy and safety of secukinumab in the treatment of moderate-to-severe plaque psoriasis: a randomized, double-blind, placebo-controlled phase II dose-ranging study. Br J Dermatol. 2013;168(2):412-21.

35. Papp K, Reich K, Leonardi CL, et al. Apremilast, an oral phosphodiesterase 4 (PDE4) inhibitor, in patients with moderate to severe plaque psoriasis: Results of a phase III, randomized, controlled trial (Efficacy and Safety Trial Evaluating the Effects of Apremilast in Psoriasis [ESTEEM] 1). J Am Acad Dermatol. 2015;73(1):37-49.

36. Papp KA, Langley RG, Lebwohl M, et al. Efficacy and safety of ustekinumab, a human interleukin-12/23 monoclonal antibody, in patients with psoriasis: 52-week results from a randomised, double-blind, placebo-controlled trial (PHOENIX 2). Lancet. 2008;371(9625):1675-84.

37. Paul C, Lacour JP, Tedremets L, et al. Efficacy, safety and usability of secukinumab administration by autoinjector/pen in psoriasis: a randomized, controlled trial (JUNCTURE). J Eur Acad Dermatol Venereol. 2015;29(6):1082-90.

38. Reich K, Nestle FO, Papp K, et al. Infliximab induction and maintenance therapy for moderate-to-severe psoriasis: a phase III, multicentre, double-blind trial. Lancet. 2005;366(9494): 1367-74.

39. Saurat JH, Stingl G, Dubertret L, et al. Efficacy and safety results from the randomized controlled comparative study of adalimumab vs. methotrexate vs. placebo in patients with psoriasis (CHAMPION). Br J Dermatol. 2007;158(3):558-66.
40. Torii H, Nakagawa H. Infliximab monotherapy in Japanese patients with moderate-to-severe plaque psoriasis and psoriatic arthritis. A randomized, double-blind, placebo-controlled multicenter trial. J Dermatol Sci. 2010;59(1):40-9.

41. Tsai T-F, Ho J-C, Song M, et al. Efficacy and safety of ustekinumab for the treatment of moderate-to-severe psoriasis: a phase III, randomized, placebo-controlled trial in Taiwanese and Korean patients (PEARL). J Dermatol Sci. 2011;63(3):154-63.

42. Tyring S, Gordon KB, Poulin Y, et al. Long-term safety and efficacy of $50 \mathrm{mg}$ of etanercept twice weekly in patients with psoriasis. Arch Dermatol. 2007;143(6):719-26.

43. Yang HZ, Wang K, Jin H-Z, et al. Infliximab monotherapy for Chinese patients with moderate to severe plaque psoriasis: a randomized, double-blind, placebo-controlled multicenter trial. Chin Med J. 2012;125(11):1845-51.

44. Zhu X, Zheng M, Song M, et al. Efficacy and safety of ustekinumab in Chinese patients with moderate to severe plaque-type psoriasis: results from a phase 3 clinical trial (LOTUS). J Drugs Dermatol. 2013;12(2):166-74.

45. Bachelez H, van de Kerkhof $P$, Strohal R, et al. Tofacitinib versus etanercept or placebo in moderate-to- severe chronic plaque psoriasis: a phase 3 randomised non-inferiority trial. Lancet. 2015;386(9993):552-61.

46. Griffiths CEM, Reich K, Lebwohl M, et al. Comparison of ixekizumab with etanercept or placebo in moderate-to-severe psoriasis (UNCOVER-2 and UNCOVER-3): results from two phase 3 randomised trials. Lancet. 2015;386(9993):541-51.

47. Gottlieb AB, Evans R, Li S, et al. Infliximab induction therapy for patients with severe plaque-type psoriasis: a randomized, double-blind, placebo-controlled trial. J Am Acad Dermatol. 2004;51(4):534-42.

48. Gordon KB, Duffin KC, Bissonnette R, et al. A phase 2 trial of guselkumab versus adalimumab for plaque psoriasis. $\mathrm{N}$ Engl $\mathrm{J}$ Med. 2015;373:136-44.

49. Gottlieb AB, Leonardi C, Kerdel F, et al. Efficacy and safety of briakinumab vs. etanercept and placebo in patients with moderate to severe chronic plaque psoriasis. $\mathrm{Br} \mathrm{J}$ Dermatol. 2011;165:652-60.

50. Papp KA, Tyring S, Lahfa M, et al. A global phase III randomized controlled trial of etanercept in psoriasis: safety, efficacy, and effect of dose reduction. Br J Dermatol. 2005;152:1304-12.

51. Strober BE, Crowley JJ, Yamauchi PS, et al. Efficacy and safety results from a phase III, randomized controlled trial comparing the safety and efficacy of briakinumab with etanercept and placebo in patients with moderate to severe chronic plaque psoriasis. Br J Dermatol. 2011;165:661-8.

52. Papp KA, Menter MA, Abe M, et al. Tofacitinib, an oral Janus kinase inhibitor, for the treatment of chronic plaque psoriasis: results from two randomized, placebo-controlled, phase III trials. Br J Dermatol. 2015;173:949-61.

53. Gordon KB, Blauvelt A, Papp KA, et al. Phase 3 trials of ixekizumab in moderate-to-severe plaque psoriasis. N Engl J Med. 2016;375:345-56.

54. Reich K, Burden AD, Eaton JN, et al. Efficacy of biologics in the treatment of moderate to severe psoriasis: a network meta-analysis of randomized controlled trials. $\mathrm{Br} \mathrm{J}$ Dermatol. 2011;166(1):179-88. 